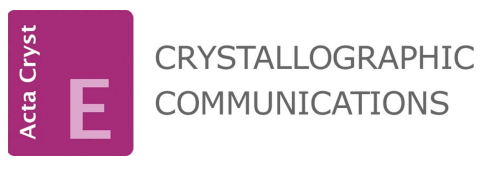

ISSN 2056-9890

Received 17 December 2017

Accepted 3 April 2018

Edited by M. Weil, Vienna University of Technology, Austria

Keywords: rhenium; fluorine; crystal structure; Raman spectroscopy; isotypism.

CCDC references: $1834616 ; 1834615$; 1834614

Supporting information: this article has supporting information at journals.iucr.org/e

\section{Syntheses, Raman spectroscopy and crystal structures of alkali hexafluoridorhenates(IV) revisited}

\author{
James Louis-Jean, ${ }^{\text {a }}$ Samundeeswari Mariappan Balasekaran, ${ }^{\text {a* }}$ Dean Smith, ${ }^{\mathrm{b}}$ Ashkan \\ Salamat, ${ }^{\mathrm{b}}$ Chien Thang Pham $^{\mathrm{c}}$ and Frederic Poineau ${ }^{\mathrm{a}}$
}

aDepartment of Chemistry, University of Nevada Las Vegas, 4505 South Maryland Parkway, Las Vegas, Nevada, 89154
United States, ${ }^{\mathbf{b}}$ Department of Physics and Astronomy and HiPSEC, University of Nevada Las Vegas, 4505 South
Maryland Parkway, Las Vegas, Nevada, 89154, United States, and ${ }^{\text {}}$ Department of Chemistry, Hanoi University of
Science, Hanoi, Vietnam. *Correspondence e-mail: m.b.eswari@unlv.edu

The $A_{2}\left[\mathrm{ReF}_{6}\right]$ ( $A=\mathrm{K}, \mathrm{Rb}$ and $\left.\mathrm{Cs}\right)$ salts are isotypic and crystallize in the trigonal space group type $P \overline{3} m 1$, adopting the $\mathrm{K}_{2}\left[\mathrm{GeF}_{6}\right]$ structure type. Common to all $A_{2}\left[\mathrm{ReF}_{6}\right]$ structures are slightly distorted octahedral $\left[\mathrm{ReF}_{6}\right]^{2-}$ anions with an average $\mathrm{Re}-\mathrm{F}$ bond length of 1.951 (8) $\AA$. In those salts, symmetry lowering on the local $\left[\mathrm{ReF}_{6}\right]^{2-}$ anions from $O_{h}$ (free anion) to $D_{3 d}$ (solid-state structure) occur. The distortions of the $\left[\mathrm{ReF}_{6}\right]^{2-}$ anions, as observed in their Raman spectra, are correlated to the size of the counter-cations.

\section{Chemical context}

The hexafluoridorhenate(IV) anion has been known for 80 years but its chemistry is understudied with respect to the heavier halogen analogs (Ruff \& Kwasnik, 1934). The scarcity of $\left[\mathrm{ReF}_{6}\right]^{2-}$ salts is attributed to the difficulties in their preparation and purification. $\mathrm{K}_{2}\left[\mathrm{ReF}_{6}\right]$ was the first hexafluoridorhenate(IV) salt to be reported; it was prepared from the solid-state melting reaction (SSMR) of $\mathrm{K}_{2}\left[\mathrm{ReBr}_{6}\right]$ with $\mathrm{KHF}_{2}$ (Ruff \& Kwasnik, 1934). Almost two decades later, ten salts comprising the $\left[\mathrm{ReF}_{6}\right]^{2-}$ anion and with different countercations $\left(\mathrm{Rb}^{+}, \mathrm{Cs}^{+}, \mathrm{PPh}_{4}{ }^{+}\left(\mathrm{Ph}=\mathrm{C}_{6} \mathrm{H}_{5}\right), \quad\left[\mathrm{Ni}\left(\mathrm{NH}_{3}\right)_{6}\right]^{2+}\right.$, $\left[\mathrm{Co}\left(\mathrm{NH}_{3}\right)_{6}\right]^{3+}, \quad\left\{\left[\mathrm{Co}\left(\mathrm{NH}_{3}\right)_{6}\right]\left(\mathrm{NO}_{3}\right)\right\}^{2+}, \quad\left\{\left[\mathrm{Cr}\left(\mathrm{NH}_{3}\right)_{6}\right]\left(\mathrm{NO}_{3}\right)\right\}^{2+}$, $\left.\left[\mathrm{Co}\left(\mathrm{NH}_{3}\right)_{5} \mathrm{Cl}\right]^{2+}, \quad\left[\mathrm{Cr}\left(\mathrm{NH}_{3}\right)_{5} \mathrm{Cl}\right]^{2+},\left[\mathrm{Co}\left(\mathrm{NH}_{3}\right)_{4}\left(\mathrm{CO}_{3}\right)\right]^{2+}\right)$ had been reported (Peacock, 1956; Weise, 1956; Pedersen et al., 2014; Brauer \& Allardt, 1962). Those salts were prepared by cation metathesis starting from $\left(\mathrm{NH}_{4}\right)_{2}\left[\mathrm{ReF}_{6}\right]$ or $\mathrm{K}_{2}\left[\mathrm{ReF}_{6}\right]$. However, the synthetic procedure to prepare $\left(\mathrm{NH}_{4}\right)_{2}\left[\mathrm{ReF}_{6}\right]$ or $\mathrm{K}_{2}\left[\mathrm{ReF}_{6}\right]$ was not explained in detail. To date, only the structures of two $\left[\mathrm{ReF}_{6}\right]^{2-}$ salts have been characterized by single crystal X-ray diffraction (SCXRD): $\mathrm{K}_{2}\left[\mathrm{ReF}_{6}\right]$ (measured at $292 \mathrm{~K}$ ) and $\left(\mathrm{PPh}_{4}\right)_{2}\left[\mathrm{ReF}_{6}\right] \cdot \mathrm{H}_{2} \mathrm{O}$ (measured at 122 K) (Clark \& Russell, 1978; Pedersen et al., 2014). Similarly, the synthesis of the $\mathrm{K}_{2}\left[\mathrm{TcF}_{6}\right]$ congener, which was reported in 1963, involves the SSMR of $\mathrm{K}_{2}\left[\mathrm{TcBr}_{6}\right]$ with $\mathrm{KHF}_{2}$ followed by an aqueous work-up (Schwochau \& Herr, 1963). However, $\left[\mathrm{TcF}_{6}\right]^{2-}$ salts have been reinvestigated recently (Balasekaran et al., 2013), and various routes for the different salts of $A_{2}\left[\mathrm{TcF}_{6}\right]\left[A=\mathrm{Na}, \mathrm{K}, \mathrm{Rb}, \mathrm{Cs}\right.$ and $\left.\mathrm{N}\left(\mathrm{CH}_{3}\right)_{4}\right]$ were reported. These salts were characterized by Raman and IR spectroscopy and by SCXRD. The $A_{2}\left[\mathrm{ReF}_{6}\right]$ salts could serve as suitable precursors to explore the chemistry of rhenium in the oxidation state IV. 
Table 1

Structural details $\left(\AA \AA^{\circ}\right)$ of the $\left[\mathrm{ReF}_{6}\right]^{2-}$ anion in this study and of the related anion in $\left[\mathrm{TcF}_{6}\right]^{2-}$ salts $^{a}$.

\begin{tabular}{lllll}
\hline & $M-\mathrm{F}, M=\mathrm{Re}$ & $\mathrm{F}-M-\mathrm{F}, M=\mathrm{Re}$ & $M-\mathrm{F}, M=\mathrm{Tc}$ & $\mathrm{F}-M-\mathrm{F}, M=\mathrm{Tc}$ \\
\hline $\mathrm{K}_{2}\left[M \mathrm{~F}_{6}\right]$ & $1.948(3)$ & $86.08(12), 93.92(12), 180$ & $1.928(6)$ & $86.93(5), 93.07(5), 180$ \\
$\mathrm{Rb}_{2}\left[M \mathrm{~F}_{6}\right]$ & $1.945(7)$ & $86.5(3), 93.5(3), 180$ & $1.933(3)$ & $87.2(2), 92.8(2), 180$ \\
$\mathrm{Cs}_{2}\left[M \mathrm{~F}_{6}\right]$ & $1.9594(18)$ & $86.86(7), 93.14(7), 180$ & $1.935(5)$ & $87.8(2), 92.2(2), 180$ \\
\hline
\end{tabular}

Note: (a) Balasekaran et al. (2013).

Here, we revisited the synthesis of $A_{2}\left[\mathrm{ReF}_{6}\right](A=\mathrm{K}, \mathrm{Rb}, \mathrm{Cs})$ salts and report their crystal structures determined from single crystal data, and their Raman spectra.

\section{Structural commentary}

The title alkaline metal salts $A_{2}\left[\mathrm{ReF}_{6}\right](A=\mathrm{K}, \mathrm{Rb}, \mathrm{Cs})$ are isotypic. They adopt the $\mathrm{K}_{2}\left[\mathrm{GeF}_{6}\right]$ structure type (Hoard \& Vincent, 1939) and crystallize in the trigonal space group type $P \overline{3} m 1$ (Table 1), just like the related $A_{2}\left[\mathrm{TcF}_{6}\right](A=\mathrm{K}, \mathrm{Rb}, \mathrm{Cs})$ compounds (Balasekaran et al., 2013). Selected bond lengths and angles of the series of $\left[\mathrm{ReF}_{6}\right]^{2-}$ anions of the present work and the reported $\left[\mathrm{TcF}_{6}\right]^{2-}$ salts (Balasekaran et al., 2013) are presented in Table 1. Representative for all other title compounds, the $\left[\mathrm{ReF}_{6}\right]^{2-}$ anion of the $\mathrm{Cs}_{2}\left[\mathrm{ReF}_{6}\right]$ salt is given in Fig. 1. The $\mathrm{Re}^{\mathrm{IV}}$ atom is located on a position with site symmetry $\overline{3} m$. (Wyckoff position $1 a$ ) at the origin of the trigonal unit cell. The six symmetry-related fluorine ligands form a slightly distorted octahedral coordination sphere around the rhenium(IV) atom. The $\mathrm{Re}-\mathrm{F}$ bond lengths for the $\mathrm{K}, \mathrm{Rb}$, and $\mathrm{Cs}$ salts of $\left[\mathrm{ReF}_{6}\right]^{2-}, 1.948$ (3), 1.945 (7) and 1.9594 (18) $\AA$, respectively, are longer than the Tc-F bond lengths for the congener $\mathrm{K}, \mathrm{Rb}$, and $\mathrm{Cs}$ salts of $\left[\mathrm{TcF}_{6}\right]^{2-}$,

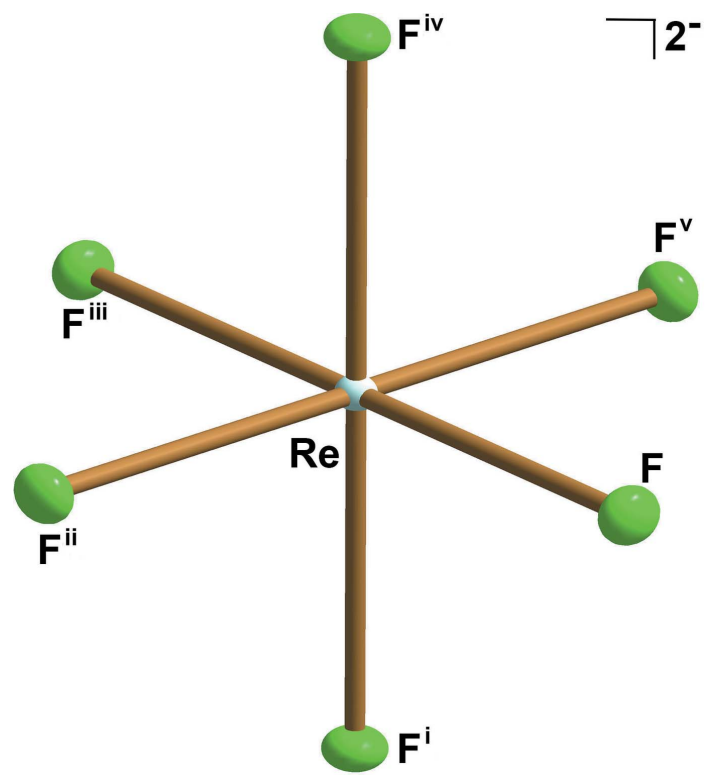

Figure 1

Representation of the $\left[\mathrm{ReF}_{6}\right]^{2-}$ anion in $\mathrm{Cs}_{2}\left[\mathrm{ReF}_{6}\right]$. Displacement ellipsoids are drawn at the $50 \%$ probability level. [Symmetry codes: (i) $-x,-y,-z$; (ii) $x-y, x,-z$; (iii) $-x+y,-x, z$; (iv) $-y, x-y, z$; (v) $y$, $-x+y,-z$.
1.928 (1), 1.933 (3), and 1.935 (5) А̊, respectively (Balasekaran et al., 2013).

In $A_{2}\left[\mathrm{ReF}_{6}\right]\left(A=\mathrm{K}^{+}, \mathrm{Rb}^{+}, \mathrm{Cs}^{+}\right)$, each cation is located on a position with site symmetry $3 m$. (Wyckoff position $2 d$ ) and is surrounded by twelve neighboring $\mathrm{F}$ atoms resulting in a $[3+6+3]$ arrangement with three groups of fluoride ligands with distances of 3.0955 (19) $\AA$ (three of such), 3.1655 (6) $\AA$ (six of such), and 3.224 (2) $\AA$ (three of such) for the $\mathrm{Cs}^{+}$salt as a representative of the three $\left[\mathrm{ReF}_{6}\right]^{2-}$ salts. These bond-length distributions are also found in the $\mathrm{K}^{+}$and $\mathrm{Rb}^{+}$salts of the $\left[\mathrm{ReF}_{6}\right]^{2-}$ complexes. This correlates well and confirms that $A_{2}\left[\mathrm{ReF}_{6}\right]$ salts are isotypic with $\mathrm{K}_{2}\left[\mathrm{GeF}_{6}\right]$ and the congener $A_{2}\left[\mathrm{TcF}_{6}\right]\left(A=\mathrm{K}^{+}, \mathrm{Rb}^{+}, \mathrm{Cs}^{+}\right)$(Balasekaran et al., 2013; Hoard \& Vincent, 1939). In comparison with the previous structure determination of $\mathrm{K}_{2}\left[\mathrm{ReF}_{6}\right]$ (Clark \& Russell, 1978), the current redetermination resulted in better reliability factors, together with a more precise determination of lattice parameters and atomic coordinates.

\section{Raman spectroscopy}

As reported previously for $\mathrm{K}_{2}\left[\mathrm{ReF}_{6}\right]$ and $A_{2}\left[\mathrm{TcF}_{6}\right](A=\mathrm{K}, \mathrm{Rb}$, Cs) (Bettinelli et al., 1987; Balasekaran et al., 2013), the $\left[\mathrm{ReF}_{6}\right]^{2-}$ anions are compressed along the crystallographic $c$ axis, thus lowering the ideal molecular symmetry of the $\left[\mathrm{ReF}_{6}\right]^{2-}$ anions from $O_{h}$ to $D_{3 d}$ in the solid state. The representive unit-cell plot of $\mathrm{Cs}_{2}\left[\mathrm{ReF}_{6}\right]$ is given in Fig. 2. The effect of symmetry lowering among the alkali metal salts of $\left[\mathrm{TcF}_{6}\right]^{2-}$ and its correlation with the vibrational spectra are

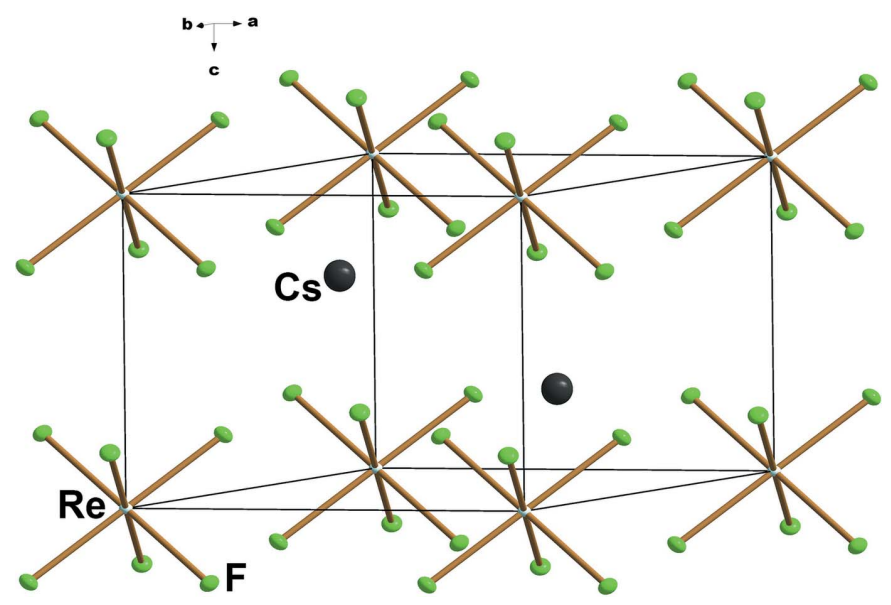

Figure 2

A packing diagram of $\mathrm{Cs}_{2}\left[\mathrm{ReF}_{6}\right]$. Displacement ellipsoids are drawn at the $50 \%$ probability level. 
Table 2

Experimental details.

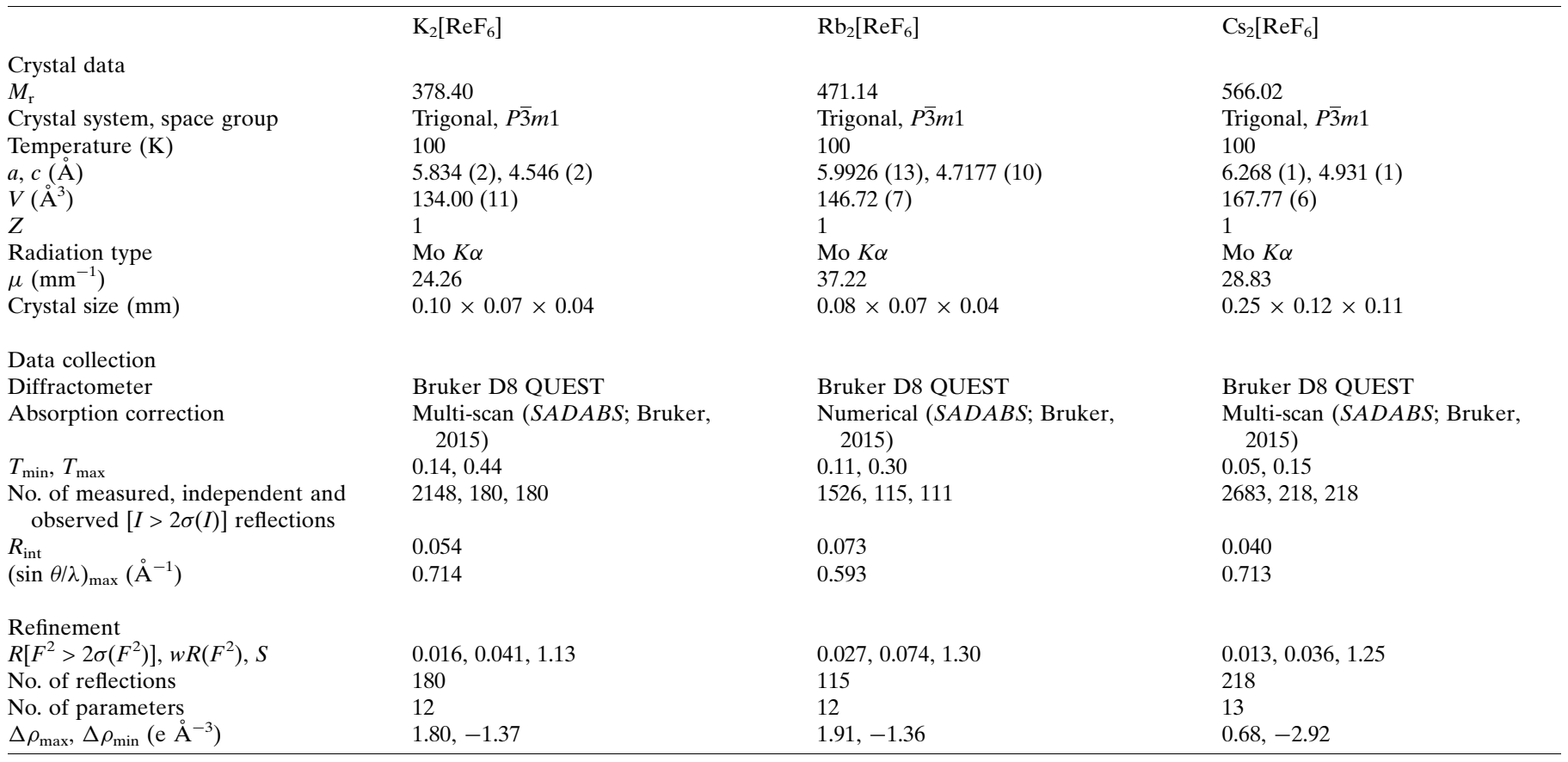

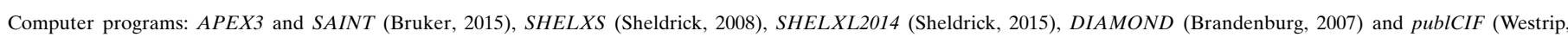
2010).

well described (Balasekaran et al., 2013). Here, a similar trend occurs for the $A_{2}\left[\mathrm{ReF}_{6}\right]$ series $(A=\mathrm{K}, \mathrm{Rb}$, Cs; Fig. 3). In the case of $\mathrm{K}_{2}\left[\mathrm{ReF}_{6}\right]$, the Raman spectrum exhibits four bands at $624,539,244$ and $224 \mathrm{~cm}^{-1}$. The latter two vibrations correspond to the $F_{2 g}$ band split due to the symmetry lowering. In the Raman spectra of $A_{2}\left[M \mathrm{~F}_{6}\right]$ complexes ( $A=\mathrm{K}, \mathrm{Rb}, \mathrm{Cs} ; M=$ $\mathrm{Tc}, \mathrm{Re})$, the $F_{2 g}$ splitting decreases from $\mathrm{K}_{2}\left[\mathrm{ReF}_{6}\right]$ to
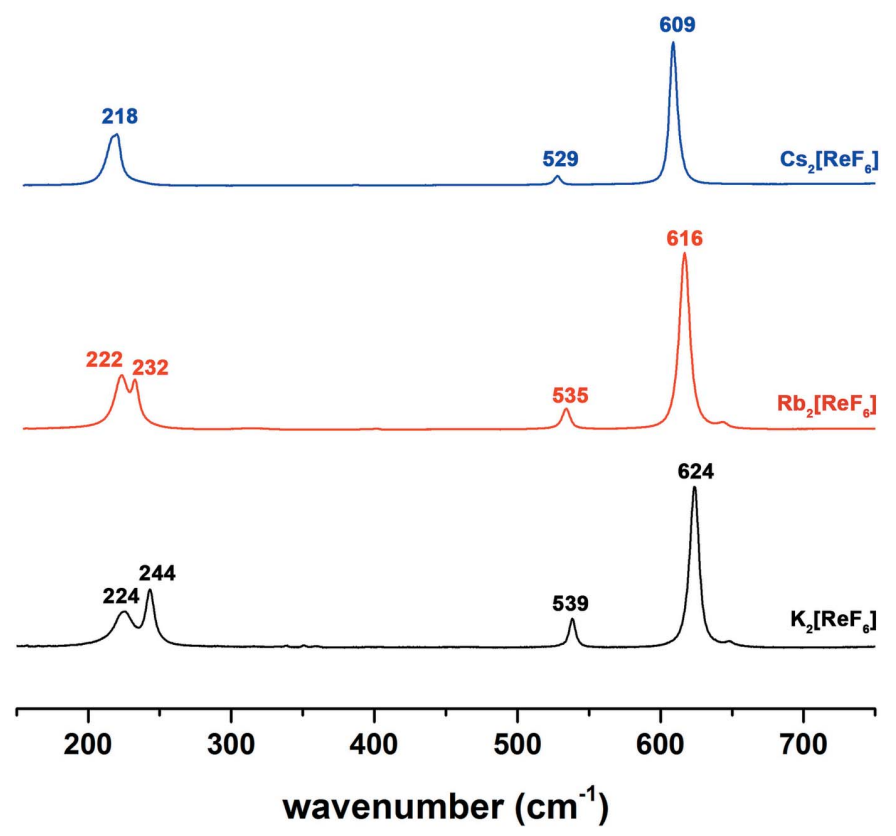

Figure 3

Raman spectra of $A_{2}\left[\mathrm{ReF}_{6}\right](A=\mathrm{K}, \mathrm{Rb}, \mathrm{Cs})$.
$\mathrm{Cs}_{2}\left[\mathrm{ReF}_{6}\right]$ due to differences in $M-\mathrm{F}$ bond length. Furthermore, the slight increase of $M-\mathrm{F}$ bond lengths from $\mathrm{K}_{2}\left[M \mathrm{~F}_{6}\right]$ to $\mathrm{Cs}_{2}\left[M \mathrm{~F}_{6}\right]$ are well represented in the Raman spectra which causes the Raman bands to shift to lower wavenumbers.

\section{Synthesis and crystallization}

Ammonium perrhenate, ammonium bifluoride, potassium fluoride, rubidium fluoride, cesium fluoride, and hydrobromic acid $(48 \%)$ were purchased from Sigma Aldrich and used without any further purification. This work was performed in a well-ventilated fume hood due to the corrosive nature of bifluoride. $\mathrm{K}_{2}\left[\mathrm{ReBr}_{6}\right]$ was prepared as described in the literature (Watt et al., 1963), and the detailed synthesis of $A_{2}\left[\mathrm{ReF}_{6}\right](A=\mathrm{K}, \mathrm{Rb}, \mathrm{Cs})$ is described below. Single crystals of $A_{2}\left[\mathrm{ReF}_{6}\right](A=\mathrm{K}, \mathrm{Rb}, \mathrm{Cs})$ were obtained by slow evaporation at room temperature of an aqueous solution of the respective salt.

\section{Synthesis of $K_{2}\left[\operatorname{ReF}_{6}\right]$}

$\mathrm{K}_{2}\left[\mathrm{ReF}_{6}\right]$ was prepared by melting $\mathrm{K}_{2}\left[\operatorname{ReBr}_{6}\right]$ (2 g, $2.69 \mathrm{mmol})$ with excess $\mathrm{KHF}_{2}(14 \mathrm{~g}, 0.18 \mathrm{~mol})$ in a nickel crucible at $673 \mathrm{~K}$ for $30 \mathrm{~min}$ in a box furnace. The resulting greyish solid product formed was allowed to cool to room temperature and was washed first with $\mathrm{MeOH}(4 \times 10 \mathrm{ml})$. Subsequently, the product was washed with several aliquots of an $\mathrm{H}_{2} \mathrm{O} / \mathrm{MeOH}$ mixture $(3 \times 5 \mathrm{ml}, 1: 4$ volume ratios $)$ and centrifuged. The pink solid obtained was dissolved in warm water $(5-10 \mathrm{ml}, 353 \mathrm{~K})$ and evaporated slowly at room temperature. The resultant pink crystals of $\mathrm{K}_{2}\left[\mathrm{ReF}_{6}\right]$ were recrystallized from warm water $(5 \mathrm{ml}, 353 \mathrm{~K})$ and colorless 
crystals of $\mathrm{K}_{2}\left[\mathrm{ReF}_{6}\right]$ were obtained. Yield: $661 \mathrm{mg}, 1.7 \mathrm{mmol}$ $(65 \%)$. IR $\left(\mathrm{KBr}, \mathrm{cm}^{-1}\right): 518,484 \mathrm{sh}(\mathrm{Re}-\mathrm{F})$.

\section{Syntheses of $A_{2}\left[\operatorname{ReF}_{6}\right](A=\mathbf{R b}, \mathrm{Cs})$ salts}

$\mathrm{K}_{2}\left[\mathrm{ReF}_{6}\right]$ (151 mg, $\left.0.4 \mathrm{mmol}\right)$ was dissolved in $4 \mathrm{ml}$ of hot water $(353 \mathrm{~K}) . M \mathrm{~F}(M=\mathrm{Rb}, \mathrm{Cs})(0.8 \mathrm{mmol})$ dissolved in $1 \mathrm{ml}$ of hot water $(353 \mathrm{~K})$ was added dropwise. The solution was allowed to evaporate slowly at room temperature. Crystals of $\mathrm{Rb}_{2}\left[\mathrm{ReF}_{6}\right]$ and $\mathrm{Cs}_{2}\left[\mathrm{ReF}_{6}\right]$ were formed in $24 \mathrm{~h}$ and washed first with cold water $(3 \times 2 \mathrm{ml})$ to remove other fluoride impurities followed by isopropanol $(3 \times 1 \mathrm{ml})$, and diethyl ether $(3 \times 1 \mathrm{ml}) . \mathrm{Rb}_{2}\left[\mathrm{ReF}_{6}\right]$ yield: $156 \mathrm{mg}, 0.33 \mathrm{mmol}(83 \%)$. IR $\left(\mathrm{KBr}, \mathrm{cm}^{-1}\right): 521(\mathrm{Re}-\mathrm{F}) . \mathrm{Cs}_{2}\left[\mathrm{ReF}_{6}\right]$ yield: $175 \mathrm{mg}$, $0.276 \mathrm{mmol}(77 \%)$. IR ( $\left.\mathrm{KBr}, \mathrm{cm}^{-1}\right)$ : 507, $480 \mathrm{sh}(\mathrm{Re}-\mathrm{F})$.

IR spectra were measured on a Shimadzu IR Affinity-1 spectrometer between 400 and $4000 \mathrm{~cm}^{-1}$. Raman spectra were recorded on a HORIBA T64000 triple spectrometer operating at $30 \mathrm{~mW}$ in subtractive mode. The spectra were taken from pure single crystals at room temperature using the $514.5 \mathrm{~nm}$ (Kr/Ar) laser line.

\section{Refinement}

Crystal data, data collection and structure refinement details are summarized in Table 2.

\section{Acknowledgements}

The authors thank Ms Julie Bertoia and Mr Charles Bynum for laboratory support, and Ms Wendee Johns for administrative support.

\section{Funding information}

Funding for this research was provided by: Department of Energy - Nuclear Science and Security Consortium (award No. DE-NA0003180).

\section{References}

Balasekaran, S. M., Molski, M., Spandl, J., Hagenbach, A., Alberto, R. \& Abram, U. (2013). Inorg. Chem. 52, 7094-7099.

Bettinelli, M., Disipio, L., Ingletto, G. \& Razzetti, C. (1987). Inorg. Chim. Acta, 133, 7-9.

Brandenburg, K. (2007). DIAMOND. Crystal Impact GbR, Bonn, Germany.

Brauer, G. \& Allardt, H. D. (1962). Z. Anorg. Allg. Chem. 316, 134140.

Bruker (2015). APEX3, SAINT and SADABS. Bruker AXS Inc., Madison, WI, USA.

Clark, G. R. \& Russell, D. R. (1978). Acta Cryst. B34, 894-895.

Hoard, J. L. \& Vincent, W. B. (1939). J. Am. Chem. Soc. 61, 28492852.

Peacock, R. D. (1956). J. Chem. Soc. pp. 1291-1293.

Pedersen, K. S., Sigrist, M., Sorensen, M. A., Barra, A. L., Weyhermuller, T., Piligkos, S., Thuesen, C. A., Vinum, M. G., Mutka, H., Weihe, H., Clerac, R. \& Bendix, J. (2014). Angew. Chem. Int. Ed. 53, 1351-1354.

Ruff, O. \& Kwasnik, W. (1934). Z. Anorg. Allg. Chem. 219, 65-81.

Schwochau, K. \& Herr, W. (1963). Angew. Chem. 75, 95.

Sheldrick, G. M. (2008). Acta Cryst. A64, 112-122.

Sheldrick, G. M. (2015). Acta Cryst. C71, 3-8.

Watt, G. W., Thompson, R. J. \& Gibbons, J. M. (1963). Inorganic Syntheses edited by J. Kleinberg, Vol 7, pp. 189-190. New York: McGraw-Hill.

Weise, E. (1956). Z. Anorg. Allg. Chem. 283, 377-389.

Westrip, S. P. (2010). J. Appl. Cryst. 43, 920-925. 


\section{supporting information}

Acta Cryst. (2018). E74, 646-649 [https://doi.org/10.1107/S2056989018005297]

Syntheses, Raman spectroscopy and crystal structures of allkali

hexafluoridorhenates(IV) revisited

James Louis-Jean, Samundeeswari Mariappan Balasekaran, Dean Smith, Ashkan Salamat, Chien

Thang Pham and Frederic Poineau

Computing details

For all structures, data collection: APEX3 (Bruker, 2015); cell refinement: SAINT (Bruker, 2015); data reduction: SAINT

(Bruker, 2015); program(s) used to solve structure: SHELXS (Sheldrick, 2008); program(s) used to refine structure:

SHELXL2014 (Sheldrick, 2015); molecular graphics: DIAMOND (Brandenburg, 2007); software used to prepare material for publication: publCIF (Westrip, 2010).

Dipotassium hexafluoridorhenate(IV) (SMB_K2ReF6_1)

Crystal data

$\mathrm{K}_{2}\left[\mathrm{ReF}_{6}\right]$

$M_{r}=378.40$

Trigonal, $P \overline{3} m 1$

$a=5.834(2) \AA$

$c=4.546(2) \AA$

$V=134.00(11) \AA^{3}$

$Z=1$

$F(000)=167$

Data collection

Bruker D8 QUEST

diffractometer

Radiation source: sealed tube, Siemens

KFFMo2K-90

Curved graphite monochromator

Detector resolution: 8.3333 pixels $\mathrm{mm}^{-1}$

$\varphi$ and $\omega$ scans

Absorption correction: multi-scan

(SADABS; Bruker, 2015)

Refinement

Refinement on $F^{2}$

Least-squares matrix: full

$R\left[F^{2}>2 \sigma\left(F^{2}\right)\right]=0.016$

$w R\left(F^{2}\right)=0.041$

$S=1.13$

180 reflections

12 parameters

0 restraints
$D_{\mathrm{x}}=4.689 \mathrm{Mg} \mathrm{m}^{-3}$

Mo $K \alpha$ radiation, $\lambda=0.71073 \AA$

Cell parameters from 2236 reflections

$\theta=4.0-30.5^{\circ}$

$\mu=24.26 \mathrm{~mm}^{-1}$

$T=100 \mathrm{~K}$

Hexagonal, translucent colourless

$0.10 \times 0.07 \times 0.04 \mathrm{~mm}$

$T_{\min }=0.14, T_{\max }=0.44$

2148 measured reflections

180 independent reflections

180 reflections with $I>2 \sigma(I)$

$R_{\text {int }}=0.054$

$\theta_{\max }=30.5^{\circ}, \theta_{\min }=4.0^{\circ}$

$h=-8 \rightarrow 8$

$k=-8 \rightarrow 8$

$l=-6 \rightarrow 6$

Primary atom site location: structure-invariant direct methods

Secondary atom site location: difference Fourier

map

$w=1 /\left[\sigma^{2}\left(F_{\mathrm{o}}{ }^{2}\right)+(0.0194 P)^{2}+0.517 P\right]$

where $P=\left(F_{\mathrm{o}}^{2}+2 F_{\mathrm{c}}^{2}\right) / 3$

$(\Delta / \sigma)_{\max }<0.001$

$\Delta \rho_{\max }=1.80 \mathrm{e}^{-3}$

$\Delta \rho_{\min }=-1.37 \mathrm{e} \AA^{-3}$ 


\section{Special details}

Geometry. All esds (except the esd in the dihedral angle between two 1.s. planes) are estimated using the full covariance matrix. The cell esds are taken into account individually in the estimation of esds in distances, angles and torsion angles; correlations between esds in cell parameters are only used when they are defined by crystal symmetry. An approximate (isotropic) treatment of cell esds is used for estimating esds involving l.s. planes.

Fractional atomic coordinates and isotropic or equivalent isotropic displacement parameters $\left(\hat{A}^{2}\right)$

\begin{tabular}{lllll}
\hline & $x$ & $y$ & $z$ & $U_{\mathrm{iso}} * / U_{\mathrm{eq}}$ \\
\hline Re1 & 0 & 0 & 0 & $0.00863(14)$ \\
F1 & $0.3254(6)$ & $0.1627(3)$ & $0.2299(6)$ & $0.0137(5)$ \\
$\mathrm{K} 1$ & 0.3333 & 0.6667 & $0.2955(4)$ & $0.0111(3)$ \\
\hline
\end{tabular}

Atomic displacement parameters $\left(\AA^{2}\right)$

\begin{tabular}{lllllll}
\hline & $U^{11}$ & $U^{22}$ & $U^{33}$ & $U^{12}$ & $U^{13}$ & $U^{23}$ \\
\hline Re1 & $0.00883(16)$ & $0.00883(16)$ & $0.0082(2)$ & $0.00441(8)$ & 0 & 0 \\
F1 & $0.0119(13)$ & $0.0162(10)$ & $0.0117(12)$ & $0.0060(6)$ & $-0.0012(10)$ & $-0.0006(5)$ \\
K1 & $0.0118(5)$ & $0.0118(5)$ & $0.0096(6)$ & $0.0059(2)$ & 0 & 0 \\
\hline
\end{tabular}

Geometric parameters $\left(\AA,{ }^{\circ}\right)$

\begin{tabular}{|c|c|c|c|}
\hline $\mathrm{Re} 1-\mathrm{F} 1$ & $1.948(3)$ & $\mathrm{F} 1-\mathrm{K} 1^{\text {viii }}$ & $2.9325(10)$ \\
\hline $\mathrm{Re} 1-\mathrm{F} 1^{\mathrm{i}}$ & $1.948(3)$ & $\mathrm{F} 1-\mathrm{K} 1^{\mathrm{vi}}$ & $2.946(3)$ \\
\hline $\mathrm{Re} 1-\mathrm{F} 1^{\mathrm{ii}}$ & $1.948(3)$ & $\mathrm{K} 1-\mathrm{F} 1^{\mathrm{xi}}$ & $2.762(3)$ \\
\hline $\mathrm{Re} 1-\mathrm{F} 1^{\mathrm{iii}}$ & $1.948(3)$ & $\mathrm{K} 1-\mathrm{F} 1^{\mathrm{x}}$ & $2.762(3)$ \\
\hline $\operatorname{Re} 1-F 1^{\text {iv }}$ & $1.948(3)$ & $\mathrm{K} 1-\mathrm{F} 1^{\mathrm{xii}}$ & $2.762(3)$ \\
\hline $\mathrm{Re} 1-\mathrm{F} 1^{\mathrm{v}}$ & $1.948(3)$ & $\mathrm{K} 1-\mathrm{F} 1^{\mathrm{xiii}}$ & $2.9325(11)$ \\
\hline $\mathrm{Re} 1-\mathrm{K} 1^{\mathrm{i}}$ & 3.6263 (13) & $\mathrm{K} 1-\mathrm{F} 1^{\mathrm{xiv}}$ & $2.9325(10)$ \\
\hline $\operatorname{Re} 1-\mathrm{K} 1^{\mathrm{vi}}$ & $3.6263(13)$ & $\mathrm{K} 1-\mathrm{F} 1^{\mathrm{iv}}$ & $2.9325(11)$ \\
\hline $\operatorname{Re} 1-\mathrm{K} 1^{\mathrm{vii}}$ & $3.6263(13)$ & $\mathrm{K} 1-\mathrm{F} 1^{\mathrm{xv}}$ & $2.9325(11)$ \\
\hline $\mathrm{Re} 1-\mathrm{K} 1$ & $3.6263(13)$ & $\mathrm{K} 1-\mathrm{F} 1^{\mathrm{xvi}}$ & $2.9325(11)$ \\
\hline $\mathrm{Re} 1-\mathrm{K} 1^{\mathrm{viii}}$ & 3.6263 (13) & $\mathrm{K} 1-\mathrm{F} 1^{\mathrm{xvii}}$ & $2.946(3)$ \\
\hline $\mathrm{Re} 1-\mathrm{K} 1^{\mathrm{ix}}$ & $3.6263(13)$ & $\mathrm{K} 1-\mathrm{F} 1^{\mathrm{ii}}$ & $2.946(3)$ \\
\hline $\mathrm{F} 1-\mathrm{K} 1^{\mathrm{x}}$ & $2.762(3)$ & $\mathrm{K} 1-\mathrm{F} 1^{\mathrm{vi}}$ & $2.946(3)$ \\
\hline $\mathrm{F} 1-\mathrm{K} 1$ & $2.9325(11)$ & & \\
\hline $\mathrm{F} 1-\mathrm{Re} 1-\mathrm{F} 1^{\mathrm{i}}$ & 180.0 & $\mathrm{~K} 1-\mathrm{F} 1-\mathrm{K} 1^{\text {viii }}$ & $168.22(13)$ \\
\hline $\mathrm{F} 1-\mathrm{Re} 1-\mathrm{F} 1^{\mathrm{ii}}$ & $86.08(12)$ & $\mathrm{Re} 1-\mathrm{F} 1-\mathrm{K} 1^{\mathrm{vi}}$ & $93.38(11)$ \\
\hline 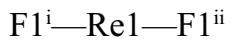 & $93.92(12)$ & $\mathrm{K} 1^{\mathrm{x}}-\mathrm{F} 1-\mathrm{K} 1^{\mathrm{vi}}$ & $105.55(10)$ \\
\hline $\mathrm{F} 1-\mathrm{Re} 1-\mathrm{F} 1^{\mathrm{iii}}$ & $93.92(12)$ & $\mathrm{K} 1-\mathrm{F} 1-\mathrm{K} 1^{\mathrm{vi}}$ & $94.27(6)$ \\
\hline $\mathrm{F} 1^{\mathrm{i}-\mathrm{Re}} 1-\mathrm{F} 1^{\mathrm{iii}}$ & $86.08(12)$ & 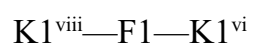 & $94.27(6)$ \\
\hline $\mathrm{F} 1^{\mathrm{ii}}-\mathrm{Re} 1-\mathrm{F} 1^{\mathrm{iii}}$ & $180.00(19)$ & 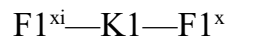 & $65.46(11)$ \\
\hline $\mathrm{F} 1-\mathrm{Re} 1-\mathrm{F} 1^{\mathrm{iv}}$ & $93.92(12)$ & $\mathrm{F} 1^{\mathrm{xi}}-\mathrm{K} 1-\mathrm{F} 1^{\mathrm{xii}}$ & $65.46(11)$ \\
\hline 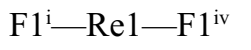 & $86.08(12)$ & $\mathrm{F} 1^{\mathrm{x}}-\mathrm{K} 1-\mathrm{F} 1^{\mathrm{xii}}$ & $65.46(11)$ \\
\hline 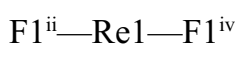 & $86.08(12)$ & $\mathrm{F} 1^{\mathrm{xi}}-\mathrm{K} 1-\mathrm{F} 1^{\mathrm{xiii}}$ & $62.44(10)$ \\
\hline 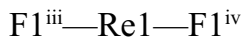 & $93.92(12)$ & $\mathrm{F} 1^{\mathrm{x}}-\mathrm{K} 1-\mathrm{F} 1^{\mathrm{xiii}}$ & $127.81(6)$ \\
\hline $\mathrm{F} 1-\operatorname{Re} 1-\mathrm{F} 1^{\mathrm{v}}$ & $86.08(12)$ & $\mathrm{F} 1^{\mathrm{xii}}-\mathrm{K} 1-\mathrm{F} 1^{\mathrm{xiii}}$ & $95.05(6)$ \\
\hline
\end{tabular}




\begin{tabular}{|c|c|}
\hline 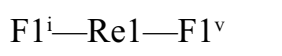 & $93.92(12)$ \\
\hline 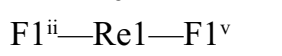 & $93.92(12)$ \\
\hline 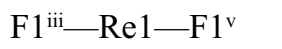 & $86.08(12)$ \\
\hline $\mathrm{F} 1^{\mathrm{iv}}-\mathrm{Re} 1-\mathrm{F} 1^{\mathrm{v}}$ & $180.00(7)$ \\
\hline $\mathrm{F} 1-\mathrm{Re} 1-\mathrm{K} 1^{\mathrm{i}}$ & $126.206(14)$ \\
\hline 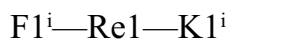 & $53.794(14)$ \\
\hline 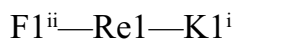 & $125.81(9)$ \\
\hline 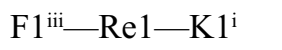 & $54.19(9)$ \\
\hline $\mathrm{F} 1^{\mathrm{iv}}-\mathrm{Re} 1-\mathrm{K} 1^{\mathrm{i}}$ & $126.205(14)$ \\
\hline $\mathrm{F} 1^{\mathrm{v}}-\mathrm{Re} 1-\mathrm{K} 1^{\mathrm{i}}$ & $53.795(14)$ \\
\hline $\mathrm{F} 1-\mathrm{Re} 1-\mathrm{K} 1^{\mathrm{vi}}$ & $54.19(9)$ \\
\hline 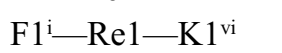 & $125.81(9)$ \\
\hline 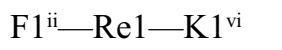 & $53.794(14)$ \\
\hline $\mathrm{F} 1^{\mathrm{iii}}-\mathrm{Re} 1-\mathrm{K} 1^{\mathrm{vi}}$ & $126.206(14)$ \\
\hline $\mathrm{F} 1^{\mathrm{iv}}-\mathrm{Re} 1-\mathrm{K} 1^{\mathrm{vi}}$ & $126.205(14)$ \\
\hline $\mathrm{F} 1^{\mathrm{v}}-\mathrm{Re} 1-\mathrm{K} 1^{\mathrm{vi}}$ & $53.795(14)$ \\
\hline $\mathrm{K} 1^{\mathrm{i}}-\operatorname{Re} 1-\mathrm{K} 1^{\mathrm{vi}}$ & $107.11(3)$ \\
\hline $\mathrm{F} 1-\mathrm{Re} 1-\mathrm{K} 1^{\mathrm{vii}}$ & $125.81(9)$ \\
\hline 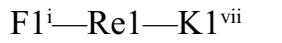 & $54.19(9)$ \\
\hline 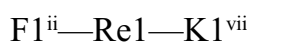 & $126.206(14)$ \\
\hline $\mathrm{F} 1^{\mathrm{iii}}-\mathrm{Re} 1-\mathrm{K} 1^{\mathrm{vii}}$ & $53.794(14)$ \\
\hline $\mathrm{F} 1^{\mathrm{iv}}-\mathrm{Re} 1-\mathrm{K} 1^{\mathrm{vii}}$ & $53.795(14)$ \\
\hline $\mathrm{F} 1^{\mathrm{v}}-\mathrm{Re} 1-\mathrm{K} 1^{\mathrm{vii}}$ & $126.205(14)$ \\
\hline $\mathrm{K} 1^{\mathrm{i}-}-\mathrm{Re} 1-\mathrm{K} 1^{\mathrm{vii}}$ & $72.89(3)$ \\
\hline 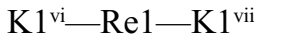 & 180.0 \\
\hline $\mathrm{F} 1-\mathrm{Re} 1-\mathrm{K} 1$ & $53.794(14)$ \\
\hline 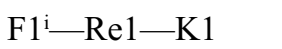 & $126.206(14)$ \\
\hline $\mathrm{F} 1^{\mathrm{ii}}-\mathrm{Re} 1-\mathrm{K} 1$ & $54.19(9)$ \\
\hline 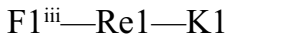 & $125.81(9)$ \\
\hline $\mathrm{F} 1^{\mathrm{iv}}-\mathrm{Re} 1-\mathrm{K} 1$ & $53.794(14)$ \\
\hline $\mathrm{F} 1{ }^{\mathrm{v}}-\mathrm{Re} 1-\mathrm{K} 1$ & $126.206(14)$ \\
\hline 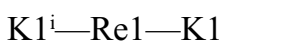 & 180.0 \\
\hline $\mathrm{K} 1^{\mathrm{vi}}-\mathrm{Re} 1-\mathrm{K} 1$ & $72.89(3)$ \\
\hline 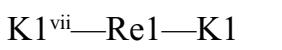 & $107.11(3)$ \\
\hline $\mathrm{F} 1-\mathrm{Re} 1-\mathrm{K} 1^{\mathrm{viii}}$ & $53.795(14)$ \\
\hline $\mathrm{F} 1^{\mathrm{i}}-\mathrm{Re} 1-\mathrm{K} 1^{\mathrm{viii}}$ & $126.205(14)$ \\
\hline $\mathrm{F} 1^{\mathrm{ii}}-\mathrm{Re} 1-\mathrm{K} 1^{\mathrm{viii}}$ & $126.206(14)$ \\
\hline 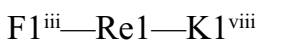 & $53.794(14)$ \\
\hline $\mathrm{F} 1^{\mathrm{iv}}-\mathrm{Re} 1-\mathrm{K} 1^{\mathrm{viii}}$ & $125.81(9)$ \\
\hline $\mathrm{F} 1^{\mathrm{v}}-\mathrm{Re} 1-\mathrm{K} 1^{\mathrm{viii}}$ & $54.19(9)$ \\
\hline $\mathrm{K} 1^{\mathrm{i}-\mathrm{Re}} 1-\mathrm{K} 1^{\mathrm{vii}}$ & $72.90(3)$ \\
\hline 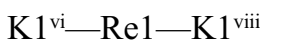 & $72.90(3)$ \\
\hline 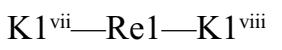 & $107.10(3)$ \\
\hline $\mathrm{K} 1-\mathrm{Re} 1-\mathrm{K} 1^{\mathrm{viii}}$ & $107.10(3)$ \\
\hline $\mathrm{F} 1-\mathrm{Re} 1-\mathrm{K} 1^{\mathrm{ix}}$ & $126.205(14)$ \\
\hline 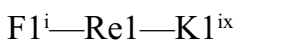 & $53.795(14)$ \\
\hline $\mathrm{F} 1^{\mathrm{ii}}-\mathrm{Re} 1-\mathrm{K} 1^{\mathrm{ix}}$ & $53.794(14)$ \\
\hline 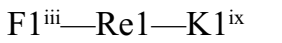 & $126.206(14)$ \\
\hline
\end{tabular}

\begin{tabular}{|c|c|}
\hline $\mathrm{F} 1^{\mathrm{xi}}-\mathrm{K} 1-\mathrm{F} 1^{\mathrm{xiv}}$ & $62.44(10)$ \\
\hline $\mathrm{F} 1^{\mathrm{x}}-\mathrm{K} 1-\mathrm{F} 1^{\mathrm{xiv}}$ & $95.05(6)$ \\
\hline 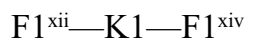 & $127.81(6)$ \\
\hline 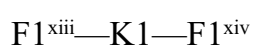 & $58.10(11)$ \\
\hline $\mathrm{F} 1^{\mathrm{xi}}-\mathrm{K} 1-\mathrm{F} 1^{\mathrm{iv}}$ & $95.05(6)$ \\
\hline $\mathrm{F} 1^{\mathrm{x}}-\mathrm{K} 1-\mathrm{F} 1^{\mathrm{iv}}$ & $127.81(6)$ \\
\hline 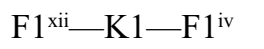 & $62.44(10)$ \\
\hline $\mathrm{F} 1^{\mathrm{xii}}-\mathrm{K} 1-\mathrm{F} 1^{\mathrm{iv}}$ & $61.22(12)$ \\
\hline $\mathrm{F} 1^{\mathrm{xiv}}-\mathrm{K} 1-\mathrm{F} 1^{\mathrm{iv}}$ & $118.98(2)$ \\
\hline $\mathrm{F} 1^{\mathrm{xi}}-\mathrm{K} 1-\mathrm{F} 1^{\mathrm{xv}}$ & $95.05(6)$ \\
\hline $\mathrm{F} 1^{\mathrm{x}}-\mathrm{K} 1-\mathrm{F} 1^{\mathrm{xv}}$ & $62.44(10)$ \\
\hline $\mathrm{F} 1^{\mathrm{xii}}-\mathrm{K} 1-\mathrm{F} 1^{\mathrm{xv}}$ & $127.81(6)$ \\
\hline 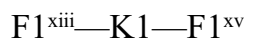 & $118.98(2)$ \\
\hline $\mathrm{F} 1^{\mathrm{xiv}}-\mathrm{K} 1-\mathrm{F} 1^{\mathrm{xv}}$ & $61.22(12)$ \\
\hline $\mathrm{F} 1^{\mathrm{iv}}-\mathrm{K} 1-\mathrm{F} 1^{\mathrm{xv}}$ & $168.22(13)$ \\
\hline $\mathrm{F} 1^{\mathrm{xi}}-\mathrm{K} 1-\mathrm{F} 1^{\mathrm{xvi}}$ & $127.81(6)$ \\
\hline $\mathrm{F} 1^{\mathrm{x}}-\mathrm{K} 1-\mathrm{F} 1^{\mathrm{xvi}}$ & $62.44(10)$ \\
\hline $\mathrm{F} 1^{\mathrm{xi}}-\mathrm{K} 1-\mathrm{F} 1^{\mathrm{xvi}}$ & $95.05(6)$ \\
\hline 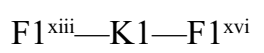 & $168.22(13)$ \\
\hline $\mathrm{F} 1^{\mathrm{xiv}}-\mathrm{K} 1-\mathrm{F} 1^{\mathrm{xvi}}$ & $118.98(2)$ \\
\hline $\mathrm{F} 1^{\mathrm{iv}}-\mathrm{K} 1-\mathrm{F} 1^{\mathrm{xvi}}$ & $118.98(2)$ \\
\hline $\mathrm{F} 1^{\mathrm{xv}}-\mathrm{K} 1-\mathrm{F} 1^{\mathrm{xvi}}$ & $58.10(11)$ \\
\hline 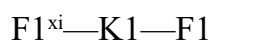 & $127.81(6)$ \\
\hline $\mathrm{F} 11^{\mathrm{x}}-\mathrm{K} 1-\mathrm{F} 1$ & $95.05(6)$ \\
\hline $\mathrm{F} 1^{\mathrm{xii}}-\mathrm{K} 1-\mathrm{F} 1$ & $62.44(10)$ \\
\hline 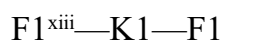 & $118.98(2)$ \\
\hline $\mathrm{F} 1^{\mathrm{xiv}}-\mathrm{K} 1-\mathrm{F} 1$ & $168.22(13)$ \\
\hline $\mathrm{F} 1^{\mathrm{iv}}-\mathrm{K} 1-\mathrm{F} 1$ & $58.09(11)$ \\
\hline $\mathrm{F} 1^{\mathrm{xv}}-\mathrm{K} 1-\mathrm{F} 1$ & $118.98(2)$ \\
\hline $\mathrm{F} 11^{\mathrm{xv}}-\mathrm{K} 1-\mathrm{F} 1$ & $61.22(11)$ \\
\hline $\mathrm{F} 1^{\mathrm{xi}}-\mathrm{K} 1-\mathrm{F} 1^{\mathrm{xvii}}$ & $105.55(10)$ \\
\hline $\mathrm{F} 1^{\mathrm{x}}-\mathrm{K} 1-\mathrm{F} 1^{\mathrm{xvii}}$ & $144.70(4)$ \\
\hline 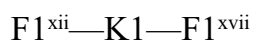 & $144.70(4)$ \\
\hline 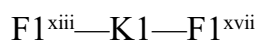 & $53.80(10)$ \\
\hline $\mathrm{F} 1^{\mathrm{xiv}}-\mathrm{K} 1-\mathrm{F} 1^{\mathrm{xvii}}$ & $53.80(10)$ \\
\hline $\mathrm{F} 1^{\mathrm{iv}}-\mathrm{K} 1-\mathrm{F} 1^{\mathrm{xvii}}$ & $85.73(6)$ \\
\hline $\mathrm{F} 1^{\mathrm{xv}}-\mathrm{K} 1-\mathrm{F} 1^{\mathrm{xvii}}$ & $85.73(6)$ \\
\hline $\mathrm{F} 1^{\mathrm{xvi}}-\mathrm{K} 1-\mathrm{F} 1^{\mathrm{xvii}}$ & $114.69(6)$ \\
\hline $\mathrm{F} 1-\mathrm{K} 1-\mathrm{F} 1^{\mathrm{xvii}}$ & $114.69(6)$ \\
\hline $\mathrm{F} 1^{\mathrm{xi}}-\mathrm{K} 1-\mathrm{F} 1^{\mathrm{ii}}$ & $144.70(4)$ \\
\hline $\mathrm{F} 1^{\mathrm{x}}-\mathrm{K} 1-\mathrm{F} 1^{\mathrm{ii}}$ & $144.70(4)$ \\
\hline 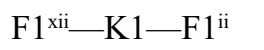 & $105.55(10)$ \\
\hline $\mathrm{F} 1^{\mathrm{xiii}}-\mathrm{K} 1-\mathrm{F} 1^{\mathrm{ii}}$ & $85.73(6)$ \\
\hline $\mathrm{F} 1^{\mathrm{xiv}}-\mathrm{K} 1-\mathrm{F} 1^{\mathrm{ii}}$ & $114.69(6)$ \\
\hline $\mathrm{F} 1^{\mathrm{iv}}-\mathrm{K} 1-\mathrm{F} 1^{\mathrm{ii}}$ & $53.80(10)$ \\
\hline $\mathrm{F} 1^{\mathrm{xv}}-\mathrm{K} 1-\mathrm{F} 1^{\mathrm{ii}}$ & $114.69(6)$ \\
\hline 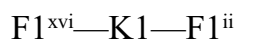 & $85.73(6)$ \\
\hline $\mathrm{F} 1-\mathrm{K} 1-\mathrm{F} 1^{\mathrm{ii}}$ & $53.80(10)$ \\
\hline
\end{tabular}




\begin{tabular}{|c|c|c|c|}
\hline $\mathrm{F} 1^{\mathrm{iv}}-\mathrm{Re} 1-\mathrm{K} 1^{\mathrm{ix}}$ & $54.19(9)$ & $\mathrm{F} 1^{\mathrm{xvii}}-\mathrm{K} 1-\mathrm{F} 1^{\mathrm{ii}}$ & $60.91(10)$ \\
\hline $\mathrm{F} 1^{\mathrm{v}}-\mathrm{Re} 1-\mathrm{K} 1^{\mathrm{ix}}$ & $125.81(9)$ & $\mathrm{F} 1^{\mathrm{xi}}-\mathrm{K} 1-\mathrm{F} 1^{\mathrm{vi}}$ & $144.70(4)$ \\
\hline $\mathrm{K} 1^{\mathrm{i}}-\mathrm{Re} 1-\mathrm{K} 1^{\mathrm{ix}}$ & $107.10(3)$ & $\mathrm{F} 1^{\mathrm{x}}-\mathrm{K} 1-\mathrm{F} 1^{\mathrm{vi}}$ & $105.55(10)$ \\
\hline 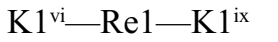 & $107.10(3)$ & $\mathrm{F} 1^{\mathrm{xii}}-\mathrm{K} 1-\mathrm{F} 1^{\mathrm{vi}}$ & $144.70(4)$ \\
\hline 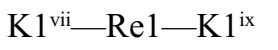 & $72.90(3)$ & $F 1^{\mathrm{xiii}}-\mathrm{K} 1-F 1^{\mathrm{vi}}$ & $114.69(6)$ \\
\hline $\mathrm{K} 1-\mathrm{Re} 1-\mathrm{K} 1^{\mathrm{ix}}$ & $72.90(3)$ & $\mathrm{F} 1^{\mathrm{xiv}}-\mathrm{K} 1-\mathrm{F} 1^{\mathrm{vi}}$ & $85.73(6)$ \\
\hline 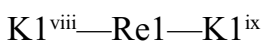 & 180.0 & $\mathrm{~F} 1^{\mathrm{iv}}-\mathrm{K} 1-\mathrm{F} 1^{\mathrm{vi}}$ & $114.69(6)$ \\
\hline $\mathrm{Re} 1-\mathrm{F} 1-\mathrm{K} 1^{\mathrm{x}}$ & $161.08(14)$ & $\mathrm{F} 1^{\mathrm{xv}}-\mathrm{K} 1-\mathrm{F} 1^{\mathrm{vi}}$ & $53.80(10)$ \\
\hline $\mathrm{Re} 1-\mathrm{F} 1-\mathrm{K} 1$ & $93.79(6)$ & $\mathrm{F} 1^{\mathrm{xvi}}-\mathrm{K} 1-\mathrm{F} 1^{\mathrm{vi}}$ & $53.80(10)$ \\
\hline $\mathrm{K} 1{ }^{\mathrm{x}}-\mathrm{F} 1-\mathrm{K} 1$ & $84.95(6)$ & $\mathrm{F} 1-\mathrm{K} 1-\mathrm{F} 1^{\mathrm{vi}}$ & $85.73(6)$ \\
\hline $\mathrm{Re} 1-\mathrm{F} 1-\mathrm{K} 1^{\mathrm{viii}}$ & $93.79(6)$ & $\mathrm{F} 1^{\mathrm{xvii}}-\mathrm{K} 1-\mathrm{F} 1^{\mathrm{vi}}$ & $60.91(10)$ \\
\hline $\mathrm{K} 1^{\mathrm{x}}-\mathrm{F} 1-\mathrm{K} 1^{\mathrm{viii}}$ & $84.95(6)$ & $\mathrm{F} 1^{\mathrm{ii}}-\mathrm{K} 1-\mathrm{F} 1^{\mathrm{vi}}$ & $60.91(10)$ \\
\hline
\end{tabular}

Symmetry codes: (i) $-x,-y,-z$; (ii) $x-y, x,-z$; (iii) $-x+y,-x, z$; (iv) $-y, x-y, z$; (v) $y,-x+y,-z$; (vi) $-x+1,-y+1,-z$; (vii) $x-1, y-1, z$; (vii) $x, y-1, z$; (ix) $-x,-y+1,-z$; (x) $-x+1,-y+1,-z+1$; (xi) $y,-x+y+1,-z+1$; (xii) $x-y, x,-z+1$; (xiii) $-x+y,-x+1, z$; (xiv) $x, y+1, z$; (xv) $-y+1, x-y+1, z$; (xvi) $-x+y+1,-x+1$, $z$; (xvii) $y,-x+y+1,-z$.

Dirubidium hexafluoridorhenate(IV) (SMB_Rb2ReF6_j)

Crystal data

$\mathrm{Rb}_{2}\left[\mathrm{ReF}_{6}\right]$

$M_{r}=471.14$

Trigonal, $P \overline{3} m 1$

$a=5.9926(13) \AA$

$c=4.7177(10) \AA$

$V=146.72(7) \AA^{3}$

$Z=1$

$F(000)=203$

\section{Data collection}

Bruker D8 QUEST

diffractometer

Radiation source: sealed tube, Siemens KFFMo2K-90

Curved graphite monochromator

Detector resolution: 8.3333 pixels $\mathrm{mm}^{-1}$

$\varphi$ and $\omega$ scans

Absorption correction: numerical

(SADABS; Bruker, 2015)

Refinement

Refinement on $F^{2}$

Least-squares matrix: full

$R\left[F^{2}>2 \sigma\left(F^{2}\right)\right]=0.027$

$w R\left(F^{2}\right)=0.074$

$S=1.30$

115 reflections

12 parameters

0 restraints
$D_{\mathrm{x}}=5.332 \mathrm{Mg} \mathrm{m}^{-3}$

Mo $K \alpha$ radiation, $\lambda=0.71073 \AA$

Cell parameters from 1612 reflections

$\theta=3.9-28.3^{\circ}$

$\mu=37.22 \mathrm{~mm}^{-1}$

$T=100 \mathrm{~K}$

Hexagonal plate, translucent colourless

$0.08 \times 0.07 \times 0.04 \mathrm{~mm}$

$T_{\min }=0.11, T_{\max }=0.30$

1526 measured reflections

115 independent reflections

111 reflections with $I>2 \sigma(I)$

$R_{\text {int }}=0.073$

$\theta_{\text {max }}=24.9^{\circ}, \theta_{\min }=3.9^{\circ}$

$h=-7 \rightarrow 7$

$k=-7 \rightarrow 7$

$l=-5 \rightarrow 5$

Primary atom site location: heavy-atom method Secondary atom site location: difference Fourier map

$w=1 /\left[\sigma^{2}\left(F_{\mathrm{o}}^{2}\right)+(0.0175 P)^{2}+3.5548 P\right]$

where $P=\left(F_{\mathrm{o}}^{2}+2 F_{\mathrm{c}}^{2}\right) / 3$

$(\Delta / \sigma)_{\max }<0.001$

$\Delta \rho_{\max }=1.91 \mathrm{e} \AA^{-3}$

$\Delta \rho_{\min }=-1.36 \mathrm{e} \AA^{-3}$ 


\section{Special details}

Geometry. All esds (except the esd in the dihedral angle between two 1.s. planes) are estimated using the full covariance matrix. The cell esds are taken into account individually in the estimation of esds in distances, angles and torsion angles; correlations between esds in cell parameters are only used when they are defined by crystal symmetry. An approximate (isotropic) treatment of cell esds is used for estimating esds involving l.s. planes.

Fractional atomic coordinates and isotropic or equivalent isotropic displacement parameters $\left(\AA^{2}\right)$

\begin{tabular}{lllll}
\hline & $x$ & $y$ & $z$ & $U_{\text {iso }} * / U_{\text {eq }}$ \\
\hline Re1 & 0 & 0 & 0 & $0.0157(5)$ \\
F1 & $0.3151(14)$ & $0.1576(7)$ & $0.2231(15)$ & $0.0151(17)$ \\
Rb1 & 0.3333 & 0.6667 & $0.2971(4)$ & $0.0138(6)$ \\
\hline
\end{tabular}

Atomic displacement parameters $\left(\AA^{2}\right)$

\begin{tabular}{lllllll}
\hline & $U^{11}$ & $U^{22}$ & $U^{33}$ & $U^{12}$ & $U^{13}$ & $U^{23}$ \\
\hline $\operatorname{Re} 1$ & $0.0133(6)$ & $0.0133(6)$ & $0.0205(8)$ & $0.0066(3)$ & 0 & 0 \\
F1 & $0.015(4)$ & $0.017(3)$ & $0.012(3)$ & $0.0077(19)$ & $0.001(3)$ & $0.0007(14)$ \\
Rb1 & $0.0131(8)$ & $0.0131(8)$ & $0.0152(12)$ & $0.0065(4)$ & 0 & 0 \\
\hline
\end{tabular}

Geometric parameters $\left(\AA,{ }^{\circ}\right)$

\begin{tabular}{|c|c|c|c|}
\hline $\mathrm{Re} 1-\mathrm{F} 1^{\mathrm{i}}$ & $1.945(7)$ & $\mathrm{F} 1-\mathrm{Rb} 1^{\mathrm{viii}}$ & $3.0181(11)$ \\
\hline $\mathrm{Re} 1-\mathrm{F} 1^{\mathrm{ii}}$ & $1.945(7)$ & $\mathrm{F} 1-\mathrm{Rb} 1^{\mathrm{vi}}$ & $3.058(7)$ \\
\hline $\mathrm{Re} 1-\mathrm{F} 1^{\mathrm{iii}}$ & $1.945(7)$ & $\mathrm{Rb} 1-\mathrm{F} 1^{\mathrm{xi}}$ & $2.907(7)$ \\
\hline $\mathrm{Re} 1-\mathrm{F} 1^{\mathrm{iv}}$ & $1.945(7)$ & $\mathrm{Rb} 1-\mathrm{F} 1^{\mathrm{x}}$ & $2.907(7)$ \\
\hline $\mathrm{Re} 1-\mathrm{F} 1^{\mathrm{v}}$ & $1.945(7)$ & $\mathrm{Rb} 1-\mathrm{F} 1^{\mathrm{xii}}$ & $2.907(7)$ \\
\hline $\mathrm{Re} 1-\mathrm{F} 1$ & $1.945(7)$ & $\mathrm{Rb} 1-\mathrm{F} 1^{\mathrm{xiii}}$ & $3.0181(11)$ \\
\hline $\mathrm{Re} 1-\mathrm{Rb} 1^{\mathrm{i}}$ & $3.7330(10)$ & $\mathrm{Rb} 1-\mathrm{F} 1^{\mathrm{xiv}}$ & $3.0181(11)$ \\
\hline $\mathrm{Re} 1-\mathrm{Rb} 1^{\mathrm{vi}}$ & $3.7330(11)$ & $\mathrm{Rb} 1-\mathrm{F} 1^{\mathrm{v}}$ & $3.0181(11)$ \\
\hline $\mathrm{Re} 1-\mathrm{Rb} 1^{\mathrm{vii}}$ & $3.7330(11)$ & $\mathrm{Rb} 1-\mathrm{F} 1^{\mathrm{xv}}$ & $3.0181(11)$ \\
\hline $\mathrm{Re} 1-\mathrm{Rb} 1$ & $3.7330(10)$ & $\mathrm{Rb} 1-\mathrm{F} 1^{\mathrm{xvi}}$ & $3.0181(11)$ \\
\hline $\mathrm{Re} 1-\mathrm{Rb} 1^{\mathrm{viii}}$ & $3.7330(11)$ & $\mathrm{Rb} 1-\mathrm{F}^{\mathrm{xvii}}$ & $3.058(7)$ \\
\hline $\operatorname{Re} 1-\mathrm{Rb} 1^{\mathrm{ix}}$ & $3.7330(11)$ & $\mathrm{Rb} 1-\mathrm{F}^{\mathrm{ii}}$ & $3.058(7)$ \\
\hline $\mathrm{F} 1-\mathrm{Rb} 1^{\mathrm{x}}$ & $2.907(7)$ & $\mathrm{Rb} 1-\mathrm{F} 1^{\mathrm{vi}}$ & $3.058(7)$ \\
\hline $\mathrm{F} 1-\mathrm{Rb} 1$ & $3.0181(11)$ & & \\
\hline 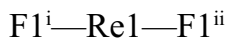 & $93.5(3)$ & $\mathrm{Rb} 1-\mathrm{F} 1-\mathrm{Rb} 1^{\mathrm{viii}}$ & $166.2(3)$ \\
\hline 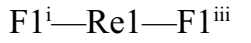 & $86.5(3)$ & $\mathrm{Re} 1-\mathrm{F} 1-\mathrm{Rb} 1^{\mathrm{vi}}$ & $93.9(2)$ \\
\hline 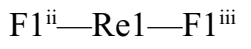 & $180.0(3)$ & $\mathrm{Rb} 1^{\mathrm{x}}-\mathrm{F} 1-\mathrm{Rb} 1^{\mathrm{vi}}$ & $104.5(2)$ \\
\hline $\mathrm{F} 1^{\mathrm{i}-}-\mathrm{Re} 1-\mathrm{F} 1^{\mathrm{iv}}$ & $93.5(3)$ & $\mathrm{Rb} 1-\mathrm{F} 1-\mathrm{Rb} 1^{\mathrm{vi}}$ & $94.26(14)$ \\
\hline $\mathrm{F} 1^{\mathrm{ii}-}-\operatorname{Re} 1-\mathrm{F} 1^{\mathrm{iv}}$ & $93.5(3)$ & 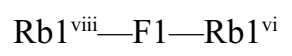 & $94.26(14)$ \\
\hline 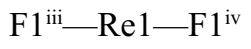 & $86.5(3)$ & $\mathrm{F} 1^{\mathrm{xi}}-\mathrm{Rb} 1-\mathrm{F} 1^{\mathrm{x}}$ & $65.8(2)$ \\
\hline 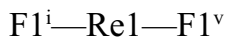 & $86.5(3)$ & $\mathrm{F} 1^{\mathrm{xi}}-\mathrm{Rb} 1-\mathrm{F} 1^{\mathrm{xii}}$ & $65.8(2)$ \\
\hline $\mathrm{F} 1^{\mathrm{ii}}-\mathrm{Re} 1-\mathrm{F} 1^{\mathrm{v}}$ & $86.5(3)$ & $\mathrm{F} 1^{\mathrm{x}}-\mathrm{Rb} 1-\mathrm{F} 1^{\mathrm{xii}}$ & $65.8(2)$ \\
\hline 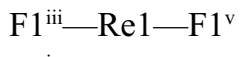 & $93.5(3)$ & 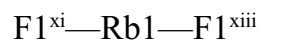 & $62.7(2)$ \\
\hline $\mathrm{F} 1^{\mathrm{iv}}-\mathrm{Re} 1-\mathrm{F} 1^{\mathrm{v}}$ & 180.0 & $\mathrm{~F} 1^{\mathrm{x}}-\mathrm{Rb} 1-\mathrm{F} 1^{\mathrm{xiii}}$ & $128.31(8)$ \\
\hline $\mathrm{F} 1{ }^{\mathrm{i}-}-\mathrm{Re} 1-\mathrm{F} 1$ & 180.0 & 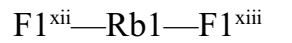 & $96.30(14)$ \\
\hline
\end{tabular}




\begin{tabular}{|c|c|c|c|}
\hline $\mathrm{F} 1{ }^{\mathrm{ii}}-\mathrm{Re} 1-\mathrm{F} 1$ & $86.5(3)$ & 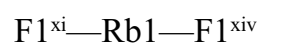 & $62.7(2)$ \\
\hline $\mathrm{F} 1^{\mathrm{iii}-\mathrm{Re} 1-\mathrm{F} 1}$ & $93.5(3)$ & $\mathrm{F} 1^{\mathrm{x}}-\mathrm{Rb} 1-\mathrm{F} 1^{\mathrm{xiv}}$ & $96.30(14)$ \\
\hline $\mathrm{F} 1^{\mathrm{iv}}-\mathrm{Re} 1-\mathrm{F} 1$ & $86.5(3)$ & 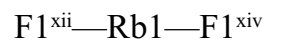 & $128.31(8)$ \\
\hline $\mathrm{F} 1^{\mathrm{v}}-\mathrm{Re} 1-\mathrm{F} 1$ & $93.5(3)$ & $\mathrm{F} 1^{\mathrm{xii}}-\mathrm{Rb} 1-\mathrm{F} 1^{\mathrm{xiv}}$ & $56.0(3)$ \\
\hline 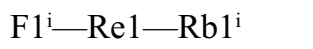 & $53.64(2)$ & $\mathrm{F} 1^{\mathrm{xi}}-\mathrm{Rb} 1-\mathrm{F} 1^{\mathrm{v}}$ & $96.30(14)$ \\
\hline 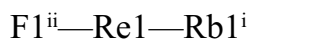 & $125.2(2)$ & $\mathrm{F} 1^{\mathrm{x}}-\mathrm{Rb} 1-\mathrm{F} 1^{\mathrm{v}}$ & $128.31(8)$ \\
\hline 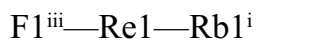 & $54.8(2)$ & 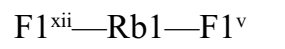 & $62.7(2)$ \\
\hline $\mathrm{F} 1^{\mathrm{iv}}-\mathrm{Re} 1-\mathrm{Rb} 1^{\mathrm{i}}$ & $53.64(2)$ & 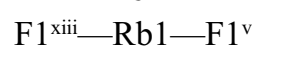 & $63.1(3)$ \\
\hline $\mathrm{F} 1^{\mathrm{v}}-\mathrm{Re} 1-\mathrm{Rb} 1^{\mathrm{i}}$ & $126.36(2)$ & $\mathrm{F} 1^{\mathrm{xiv}}-\mathrm{Rb} 1-\mathrm{F} 1^{\mathrm{v}}$ & $118.68(6)$ \\
\hline $\mathrm{F} 1-\mathrm{Re} 1-\mathrm{Rb} 1^{\mathrm{i}}$ & $126.36(2)$ & 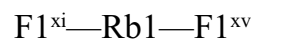 & $96.30(14)$ \\
\hline $\mathrm{F} 1^{\mathrm{i}-}-\mathrm{Re} 1-\mathrm{Rb} 1^{\mathrm{vi}}$ & $125.2(2)$ & $\mathrm{F} 1^{\mathrm{x}}-\mathrm{Rb} 1-\mathrm{F} 1^{\mathrm{xv}}$ & $62.7(2)$ \\
\hline $\mathrm{F} 1^{\mathrm{ii}}-\mathrm{Re} 1-\mathrm{Rb} 1^{\mathrm{vi}}$ & $53.64(2)$ & 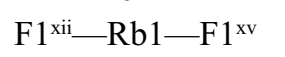 & $128.31(8)$ \\
\hline 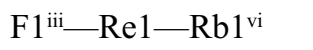 & $126.36(2)$ & 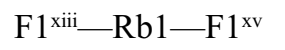 & $118.68(5)$ \\
\hline $\mathrm{F} 1^{\mathrm{iv}}-\mathrm{Re} 1-\mathrm{Rb} 1^{\mathrm{vi}}$ & $53.64(2)$ & $\mathrm{F} 1^{\mathrm{xiv}}-\mathrm{Rb} 1-F 1^{\mathrm{xv}}$ & $63.1(3)$ \\
\hline $\mathrm{F} 1^{\mathrm{v}}-\mathrm{Re} 1-\mathrm{Rb} 1^{\mathrm{vi}}$ & $126.36(2)$ & $\mathrm{F} 1^{\mathrm{v}}-\mathrm{Rb} 1-\mathrm{F} 1^{\mathrm{xv}}$ & $166.2(3)$ \\
\hline $\mathrm{F} 1-\mathrm{Re} 1-\mathrm{Rb} 1^{\mathrm{vi}}$ & $54.8(2)$ & 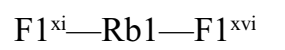 & $128.31(8)$ \\
\hline $\mathrm{Rb} 1^{\mathrm{i}}-\mathrm{Re} 1-\mathrm{Rb}^{\mathrm{vi}}$ & $106.77(3)$ & $\mathrm{F} 1^{\mathrm{x}}-\mathrm{Rb} 1-\mathrm{F} 1^{\mathrm{xvi}}$ & $62.7(2)$ \\
\hline 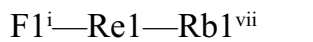 & $54.8(2)$ & $\mathrm{F} 1^{\mathrm{xi}}-\mathrm{Rb} 1-\mathrm{F} 1^{\mathrm{xvi}}$ & $96.30(14)$ \\
\hline 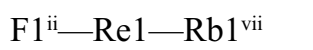 & $126.36(2)$ & $\mathrm{F} 1^{\mathrm{xii}}-\mathrm{Rb} 1-\mathrm{F} 1^{\mathrm{xvi}}$ & $166.2(3)$ \\
\hline 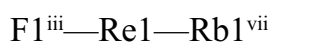 & $53.64(2)$ & $\mathrm{F} 1^{\mathrm{xiv}}-\mathrm{Rb} 1-\mathrm{F} 1^{\mathrm{xvi}}$ & $118.68(6)$ \\
\hline $\mathrm{F} 1^{\mathrm{iv}}-\mathrm{Re} 1-\mathrm{Rb} 1^{\mathrm{vi}}$ & $126.36(2)$ & $\mathrm{F} 1^{\mathrm{v}}-\mathrm{Rb} 1-\mathrm{F} 1^{\mathrm{xvi}}$ & $118.68(5)$ \\
\hline $\mathrm{F} 1^{\mathrm{v}}-\mathrm{Re} 1-\mathrm{Rb} 1^{\mathrm{vii}}$ & $53.64(2)$ & $\mathrm{F} 1^{\mathrm{xv}}-\mathrm{Rb} 1-\mathrm{F} 1^{\mathrm{xvi}}$ & $56.0(3)$ \\
\hline $\mathrm{F} 1-\operatorname{Re} 1-\mathrm{Rb} 1^{\mathrm{vii}}$ & $125.2(2)$ & 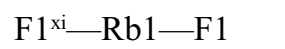 & $128.31(8)$ \\
\hline $\mathrm{Rb} 1^{\mathrm{i}}-\mathrm{Re} 1-\mathrm{Rb} 1^{\mathrm{vii}}$ & $73.23(3)$ & $\mathrm{F} 1{ }^{\mathrm{x}}-\mathrm{Rb} 1-\mathrm{F} 1$ & $96.30(14)$ \\
\hline 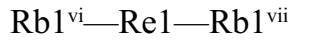 & 180.0 & 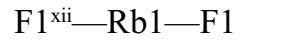 & $62.7(2)$ \\
\hline 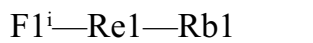 & $126.36(2)$ & $\mathrm{F} 1^{\mathrm{xii}}-\mathrm{Rb} 1-\mathrm{F} 1$ & $118.68(6)$ \\
\hline 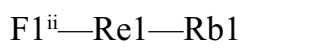 & $54.8(2)$ & $\mathrm{F} 1^{\mathrm{xiv}}-\mathrm{Rb} 1-\mathrm{F} 1$ & $166.2(3)$ \\
\hline 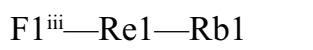 & $125.2(2)$ & 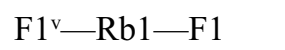 & $56.0(3)$ \\
\hline $\mathrm{F} 1^{\mathrm{iv}}-\mathrm{Re} 1-\mathrm{Rb} 1$ & $126.36(2)$ & $\mathrm{F} 1^{\mathrm{xv}}-\mathrm{Rb} 1-\mathrm{F} 1$ & $118.68(5)$ \\
\hline $\mathrm{F} 1^{\mathrm{v}}-\mathrm{Re} 1-\mathrm{Rb} 1$ & $53.64(2)$ & $\mathrm{F} 1^{\mathrm{xvi}}-\mathrm{Rb} 1-\mathrm{F} 1$ & $63.1(3)$ \\
\hline $\mathrm{F} 1-\mathrm{Re} 1-\mathrm{Rb} 1$ & $53.64(2)$ & 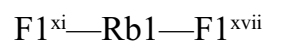 & $104.5(2)$ \\
\hline $\mathrm{Rb} 1 \mathrm{i}-\mathrm{Re} 1-\mathrm{Rb} 1$ & 180.0 & $\mathrm{~F} 1^{\mathrm{x}}-\mathrm{Rb} 1-\mathrm{F} 1^{\mathrm{xvii}}$ & $144.30(9)$ \\
\hline 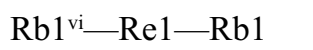 & $73.23(3)$ & $\mathrm{F} 1^{\mathrm{xii}}-\mathrm{Rb} 1-\mathrm{F} 1^{\mathrm{xvi}}$ & $144.30(9)$ \\
\hline 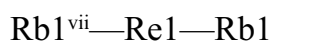 & $106.77(3)$ & $\mathrm{F} 1^{\mathrm{xii}}-\mathrm{Rb} 1-\mathrm{F} 1^{\mathrm{xvii}}$ & $52.0(2)$ \\
\hline 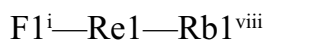 & $126.36(2)$ & $\mathrm{F} 1^{\mathrm{xiv}}-\mathrm{Rb} 1-\mathrm{F} 1^{\mathrm{xvii}}$ & $52.0(2)$ \\
\hline $\mathrm{F} 1^{\mathrm{ii}}-\mathrm{Re} 1-\mathrm{Rb} 1^{\mathrm{viii}}$ & $126.36(2)$ & $\mathrm{F} 1^{\mathrm{v}}-\mathrm{Rb} 1-\mathrm{F} 1^{\mathrm{xvii}}$ & $85.74(14)$ \\
\hline 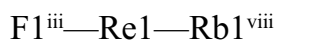 & $53.64(2)$ & $\mathrm{F} 1^{\mathrm{xv}}-\mathrm{Rb} 1-\mathrm{F} 1^{\mathrm{xvii}}$ & $85.74(14)$ \\
\hline $\mathrm{F} 1^{\mathrm{iv}}-\mathrm{Re} 1-\mathrm{Rb} 1^{\mathrm{viii}}$ & $54.8(2)$ & $\mathrm{F} 1^{\mathrm{xvi}}-\mathrm{Rb} 1-\mathrm{F} 1^{\mathrm{xvii}}$ & $114.25(9)$ \\
\hline $\mathrm{F} 1^{\mathrm{v}}-\mathrm{Re} 1-\mathrm{Rb} 1^{\mathrm{viii}}$ & $125.2(2)$ & $\mathrm{F} 1-\mathrm{Rb} 1-\mathrm{F} 1^{\mathrm{xvii}}$ & $114.25(9)$ \\
\hline $\mathrm{F} 1-\mathrm{Re} 1-\mathrm{Rb} 1^{\mathrm{viii}}$ & $53.64(2)$ & $\mathrm{F} 1^{\mathrm{xi}}-\mathrm{Rb} 1-\mathrm{F} 1^{\mathrm{ii}}$ & $144.30(9)$ \\
\hline 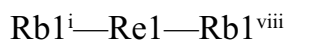 & $73.23(3)$ & $\mathrm{F} 1^{\mathrm{x}}-\mathrm{Rb} 1-\mathrm{F} 1^{\mathrm{ii}}$ & $144.30(9)$ \\
\hline $\mathrm{Rb} 1^{\mathrm{vi}}-\mathrm{Re} 1-\mathrm{Rb} 1^{\mathrm{viii}}$ & $73.23(3)$ & $\mathrm{F} 1^{\mathrm{xii}}-\mathrm{Rb} 1-\mathrm{F} 1^{\mathrm{ii}}$ & $104.5(2)$ \\
\hline 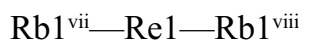 & $106.77(3)$ & $\mathrm{F} 1^{\mathrm{xiii}}-\mathrm{Rb} 1-\mathrm{F} 1^{\mathrm{ii}}$ & $85.74(14)$ \\
\hline $\mathrm{Rb} 1-\mathrm{Re} 1-\mathrm{Rb} 1^{\mathrm{viii}}$ & $106.77(3)$ & $\mathrm{F} 1^{\mathrm{xiv}}-\mathrm{Rb} 1-\mathrm{F} 1^{\mathrm{ii}}$ & $114.25(9)$ \\
\hline 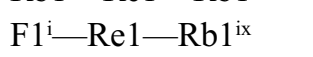 & $53.64(2)$ & $\mathrm{F} 1^{\mathrm{v}}-\mathrm{Rb} 1-\mathrm{F} 1^{\mathrm{ii}}$ & $52.0(2)$ \\
\hline $\mathrm{F} 1^{\mathrm{ii}}-\mathrm{Re} 1-\mathrm{Rb} 1^{\mathrm{ix}}$ & $53.64(2)$ & $\mathrm{F} 1^{\mathrm{xv}}-\mathrm{Rb} 1-\mathrm{F} 1^{\mathrm{ii}}$ & $114.25(9)$ \\
\hline 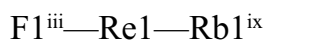 & $126.36(2)$ & $\mathrm{F} 1^{\mathrm{xvi}}-\mathrm{Rb} 1-\mathrm{F} 1^{\mathrm{ii}}$ & $85.74(14)$ \\
\hline $\mathrm{F} 1^{\mathrm{iv}}-\mathrm{Re} 1-\mathrm{Rb} 1^{\mathrm{ix}}$ & $125.2(2)$ & $\mathrm{F} 1-\mathrm{Rb} 1-\mathrm{F} 1^{\mathrm{ii}}$ & $52.0(2)$ \\
\hline
\end{tabular}




\begin{tabular}{|c|c|c|c|}
\hline $\mathrm{F} 1^{\mathrm{v}}-\mathrm{Re} 1-\mathrm{Rb} 1^{\mathrm{ix}}$ & $54.8(2)$ & $\mathrm{F} 1^{\mathrm{xvii}}-\mathrm{Rb} 1-\mathrm{F} 1^{\mathrm{ii}}$ & $62.2(2)$ \\
\hline $\mathrm{F} 1-\operatorname{Re} 1-\mathrm{Rb} 1^{\mathrm{ix}}$ & $126.36(2)$ & $\mathrm{F} 1^{\mathrm{xi}}-\mathrm{Rb} 1-\mathrm{F} 1^{\mathrm{vi}}$ & $144.30(9)$ \\
\hline 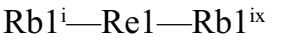 & $106.77(3)$ & $\mathrm{F} 1^{\mathrm{x}}-\mathrm{Rb} 1-\mathrm{F} 1^{\mathrm{vi}}$ & $104.5(2)$ \\
\hline 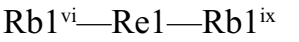 & $106.77(3)$ & 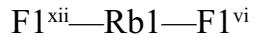 & $144.30(9)$ \\
\hline $\mathrm{Rb} 1^{\mathrm{vii}}-\mathrm{Re} 1-\mathrm{Rb} 1^{\mathrm{ix}}$ & $73.23(3)$ & $\mathrm{F} 1^{\mathrm{xii}}-\mathrm{Rb} 1-\mathrm{F} 1^{\mathrm{vi}}$ & $114.25(9)$ \\
\hline $\mathrm{Rb} 1-\mathrm{Re} 1-\mathrm{Rb} 1^{\mathrm{ix}}$ & $73.23(3)$ & $\mathrm{F} 1^{\mathrm{xiv}}-\mathrm{Rb} 1-\mathrm{F} 1^{\mathrm{vi}}$ & $85.74(14)$ \\
\hline $\mathrm{Rb} 1^{\text {viii }-R e 1-R b 1^{i x}}$ & 180.0 & $\mathrm{~F} 1^{\mathrm{v}}-\mathrm{Rb} 1-\mathrm{F} 1^{\mathrm{vi}}$ & $114.25(9)$ \\
\hline $\mathrm{Re} 1-\mathrm{F} 1-\mathrm{Rb} 1^{\mathrm{x}}$ & $161.6(3)$ & $\mathrm{F} 1^{\mathrm{xv}}-\mathrm{Rb} 1-\mathrm{F} 1^{\mathrm{vi}}$ & $52.0(2)$ \\
\hline $\mathrm{Re} 1-\mathrm{F} 1-\mathrm{Rb} 1$ & $95.10(14)$ & 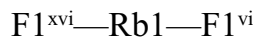 & $52.0(2)$ \\
\hline $\mathrm{Rb} 1^{\mathrm{x}}-\mathrm{F} 1-\mathrm{Rb} 1$ & $83.70(14)$ & $\mathrm{F} 1-\mathrm{Rb} 1-\mathrm{F} 1^{\mathrm{vi}}$ & $85.74(14)$ \\
\hline $\mathrm{Re} 1-\mathrm{F} 1-\mathrm{Rb} 1^{\mathrm{viii}}$ & $95.10(14)$ & 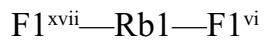 & $62.2(2)$ \\
\hline $\mathrm{Rb} 1^{\mathrm{x}}-\mathrm{F} 1-\mathrm{Rb} 1^{\text {viii }}$ & $83.70(14)$ & 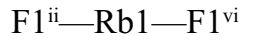 & $62.2(2)$ \\
\hline
\end{tabular}

Symmetry codes: (i) $-x,-y,-z$; (ii) $x-y, x,-z$; (iii) $-x+y,-x, z$; (iv) $y,-x+y,-z$; (v) $-y, x-y, z$; (vi) $-x+1,-y+1,-z$; (vii) $x-1, y-1, z$; (viii) $x, y-1, z$; (ix) $-x,-y+1,-z$; (x) $-x+1,-y+1,-z+1$; (xi) $y,-x+y+1,-z+1$; (xii) $x-y, x,-z+1$; (xii) $-x+y,-x+1, z$; (xiv) $x, y+1, z$; (xv) $-y+1, x-y+1, z$; (xvi) $-x+y+1,-x+1$, $z$; (xvii) $y,-x+y+1,-z$.

Dicaesium hexafluoridorhenate(IV) (SMB_Cs2ReF6_1a)

Crystal data

$\mathrm{Cs}_{2}\left[\mathrm{ReF}_{6}\right]$

$M_{r}=566.02$

Trigonal, $P \overline{3} \mathrm{~m} 1$

$a=6.268(1) \AA$

$c=4.931(1) \AA$

$V=167.77(6) \AA^{3}$

$Z=1$

$F(000)=239$

\section{Data collection}

Bruker D8 QUEST

diffractometer

Radiation source: sealed tube, Siemens KFFMo2K-90

Curved graphite monochromator

Detector resolution: 8.3333 pixels $\mathrm{mm}^{-1}$

$\varphi$ and $\omega$ scans

Absorption correction: multi-scan

(SADABS; Bruker, 2015)

\section{Refinement}

Refinement on $F^{2}$

Least-squares matrix: full

$R\left[F^{2}>2 \sigma\left(F^{2}\right)\right]=0.013$

$w R\left(F^{2}\right)=0.036$

$S=1.25$

218 reflections

13 parameters

0 restraints

Primary atom site location: structure-invariant direct methods
$D_{\mathrm{x}}=5.602 \mathrm{Mg} \mathrm{m}^{-3}$

Mo $K \alpha$ radiation, $\lambda=0.71073 \AA$

Cell parameters from 135 reflections

$\theta=3.8-32.6^{\circ}$

$\mu=28.83 \mathrm{~mm}^{-1}$

$T=100 \mathrm{~K}$

Hexagonal plate, clear colourless

$0.25 \times 0.12 \times 0.11 \mathrm{~mm}$

$T_{\min }=0.05, T_{\max }=0.15$

2683 measured reflections

218 independent reflections

218 reflections with $I>2 \sigma(I)$

$R_{\text {int }}=0.040$

$\theta_{\text {max }}=30.4^{\circ}, \theta_{\min }=3.8^{\circ}$

$h=-8 \rightarrow 8$

$k=-8 \rightarrow 8$

$l=-7 \rightarrow 7$

Secondary atom site location: difference Fourier

$$
\begin{gathered}
\text { map } \\
w=1 /\left[\sigma^{2}\left(F_{\mathrm{o}}^{2}\right)+(0.0\right. \\
\text { where } P=\left(F_{\mathrm{o}}^{2}+\right. \\
(\Delta / \sigma)_{\max }<0.001 \\
\Delta \rho_{\max }=0.68 \mathrm{e} \AA^{-3} \\
\Delta \rho_{\min }=-2.92 \mathrm{e} \AA^{-3}
\end{gathered}
$$$$
w=1 /\left[\sigma^{2}\left(F_{\mathrm{o}}^{2}\right)+(0.0181 P)^{2}+0.1419 P\right]
$$$$
\text { where } P=\left(F_{\mathrm{o}}^{2}+2 F_{\mathrm{c}}^{2}\right) / 3
$$

Extinction correction: SHELXL2014

(Sheldrick, 2015)

Extinction coefficient: 0.029 (2) 


\section{Special details}

Geometry. All esds (except the esd in the dihedral angle between two 1.s. planes) are estimated using the full covariance matrix. The cell esds are taken into account individually in the estimation of esds in distances, angles and torsion angles; correlations between esds in cell parameters are only used when they are defined by crystal symmetry. An approximate (isotropic) treatment of cell esds is used for estimating esds involving l.s. planes.

Fractional atomic coordinates and isotropic or equivalent isotropic displacement parameters $\left(\AA^{2}\right)$

\begin{tabular}{lllll}
\hline & $x$ & $y$ & $z$ & $U_{\mathrm{iso}} * / U_{\mathrm{eq}}$ \\
\hline $\operatorname{Re} 1$ & 0 & 0 & 0 & $0.00427(15)$ \\
F1 & $0.3027(3)$ & $0.15135(17)$ & $0.2165(4)$ & $0.0092(4)$ \\
Cs1 & 0.3333 & 0.6667 & $0.30027(9)$ & $0.00615(14)$ \\
\hline
\end{tabular}

Atomic displacement parameters $\left(\mathscr{A}^{2}\right)$

\begin{tabular}{lllllll}
\hline & $U^{11}$ & $U^{22}$ & $U^{33}$ & $U^{12}$ & $U^{13}$ & $U^{23}$ \\
\hline Re1 & $0.00483(16)$ & $0.00483(16)$ & $0.0032(2)$ & $0.00241(8)$ & 0 & 0 \\
F1 & $0.0088(8)$ & $0.0107(6)$ & $0.0075(9)$ & $0.0044(4)$ & $-0.0023(6)$ & $-0.0012(3)$ \\
Cs1 & $0.00661(16)$ & $0.00661(16)$ & $0.0052(2)$ & $0.00331(8)$ & 0 & 0 \\
\hline
\end{tabular}

Geometric parameters $\left(\AA,{ }^{\circ}\right)$

\begin{tabular}{|c|c|c|c|}
\hline $\mathrm{Re} 1-\mathrm{F} 1^{\mathrm{i}}$ & $1.9594(18)$ & $\mathrm{F} 1-\mathrm{Cs} 1^{\text {viii }}$ & $3.1655(6)$ \\
\hline $\mathrm{Re} 1-\mathrm{F} 1^{\mathrm{ii}}$ & $1.9594(18)$ & $\mathrm{F} 1-\mathrm{Cs} 1^{\mathrm{vi}}$ & $3.224(2)$ \\
\hline $\mathrm{Re} 1-\mathrm{F} 1^{\mathrm{iii}}$ & $1.9594(18)$ & $\mathrm{Cs} 1-\mathrm{F} 1^{\mathrm{xi}}$ & $3.0955(19)$ \\
\hline $\mathrm{Re} 1-\mathrm{F} 1^{\mathrm{iv}}$ & $1.9594(18)$ & $\mathrm{Cs} 1-\mathrm{F} 1^{\mathrm{x}}$ & $3.0955(19)$ \\
\hline $\mathrm{Re} 1-\mathrm{F} 1^{\mathrm{v}}$ & $1.9594(18)$ & $\mathrm{Cs} 1-\mathrm{F} 1^{\mathrm{xii}}$ & $3.0955(19)$ \\
\hline $\mathrm{Re} 1-\mathrm{F} 1$ & $1.9594(18)$ & $\mathrm{Cs} 1-\mathrm{F} 1^{\mathrm{xiii}}$ & $3.1655(6)$ \\
\hline $\operatorname{Re} 1-\operatorname{Cs} 1^{i}$ & $3.9100(6)$ & $\mathrm{Cs} 1-\mathrm{F} 1^{\mathrm{xiv}}$ & $3.1655(6)$ \\
\hline $\mathrm{Re} 1-\mathrm{Cs} 1^{\mathrm{vi}}$ & $3.9100(6)$ & $\mathrm{Cs} 1-\mathrm{F} 1^{\mathrm{iii}}$ & $3.1655(6)$ \\
\hline $\operatorname{Re} 1-\mathrm{Cs}^{1 \mathrm{vi}}$ & $3.9100(6)$ & $\mathrm{Cs} 1-\mathrm{F} 1^{\mathrm{xv}}$ & $3.1655(6)$ \\
\hline $\mathrm{Re} 1-\mathrm{Cs} 1$ & $3.9100(6)$ & $\mathrm{Cs} 1-\mathrm{F} 1^{\mathrm{xvi}}$ & $3.1655(6)$ \\
\hline $\operatorname{Re} 1-\mathrm{Cs} 1^{\text {viii }}$ & $3.9100(6)$ & $\mathrm{Cs} 1-\mathrm{F} 1^{\mathrm{xvii}}$ & $3.224(2)$ \\
\hline $\operatorname{Re} 1-\operatorname{Cs} 1^{\text {ix }}$ & $3.9100(6)$ & $\mathrm{Cs} 1-\mathrm{F}^{\mathrm{iv}}$ & $3.224(2)$ \\
\hline $\mathrm{F} 1-\mathrm{Cs} 1^{\mathrm{x}}$ & 3.0955 (19) & $\mathrm{Cs} 1-\mathrm{F} 1^{\mathrm{vi}}$ & $3.224(2)$ \\
\hline $\mathrm{F} 1-\mathrm{Cs} 1$ & $3.1655(6)$ & & \\
\hline 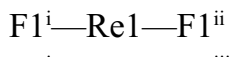 & $93.14(7)$ & $\mathrm{Cs} 1-\mathrm{F} 1-\mathrm{Cs} 1^{\mathrm{viii}}$ & $163.82(6)$ \\
\hline 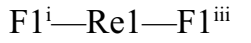 & $86.86(7)$ & $\mathrm{Re} 1-\mathrm{F} 1-\mathrm{Cs} 1^{\mathrm{vi}}$ & $94.78(7)$ \\
\hline 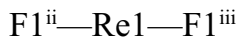 & $180.00(4)$ & $\mathrm{Cs} 1^{\mathrm{x}}-\mathrm{F} 1-\mathrm{Cs} 1^{\mathrm{vi}}$ & $102.55(5)$ \\
\hline $\mathrm{F} 1^{\mathrm{i}-}-\mathrm{Re} 1-\mathrm{F} 1^{\mathrm{iv}}$ & $93.14(7)$ & $\mathrm{Cs} 1-\mathrm{F} 1-\mathrm{Cs} 1^{\mathrm{vi}}$ & $94.07(3)$ \\
\hline $\mathrm{F} 1^{\mathrm{ii}-}-\operatorname{Re} 1-\mathrm{F} 1^{\mathrm{iv}}$ & $93.14(7)$ & $\mathrm{Cs} 1^{\mathrm{viii}}-\mathrm{F} 1-\mathrm{Cs} 1^{\mathrm{vi}}$ & $94.07(3)$ \\
\hline 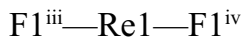 & $86.86(7)$ & $\mathrm{F} 1^{\mathrm{xi}}-\mathrm{Cs} 1-\mathrm{F} 1^{\mathrm{x}}$ & $67.11(6)$ \\
\hline 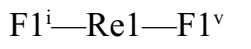 & $86.86(7)$ & $\mathrm{F} 1^{\mathrm{xi}}-\mathrm{Cs} 1-\mathrm{F} 1^{\mathrm{xii}}$ & $67.11(6)$ \\
\hline $\mathrm{F} 1^{\mathrm{ii}}-\mathrm{Re} 1-\mathrm{F} 1^{\mathrm{v}}$ & $86.86(7)$ & $\mathrm{F} 1^{\mathrm{x}}-\mathrm{Cs} 1-\mathrm{F} 1^{\mathrm{xii}}$ & $67.11(6)$ \\
\hline 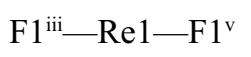 & $93.14(7)$ & $\mathrm{F} 1^{\mathrm{xi}}-\mathrm{Cs} 1-\mathrm{F} 1^{\mathrm{xiii}}$ & $62.38(6)$ \\
\hline $\mathrm{F} 1^{\mathrm{iv}}-\mathrm{Re} 1-\mathrm{F} 1^{\mathrm{v}}$ & $180.00(8)$ & $\mathrm{F} 1^{\mathrm{x}}-\mathrm{Cs} 1-\mathrm{F} 1^{\mathrm{xiii}}$ & $129.122(17)$ \\
\hline $\mathrm{F} 1{ }^{\mathrm{i}-}-\mathrm{Re} 1-\mathrm{F} 1$ & 180.0 & $\mathrm{~F} 1^{\mathrm{xii}}-\mathrm{Cs} 1-\mathrm{F} 1^{\mathrm{xiii}}$ & $97.70(3)$ \\
\hline
\end{tabular}




\begin{tabular}{|c|c|}
\hline $\mathrm{F} 1{ }^{\mathrm{ii}}-\mathrm{Re} 1-\mathrm{F} 1$ & $86.86(7)$ \\
\hline 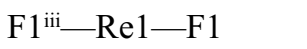 & $93.14(7)$ \\
\hline $\mathrm{F} 1^{\mathrm{iv}}-\mathrm{Re} 1-\mathrm{F} 1$ & $86.86(7)$ \\
\hline $\mathrm{F} 1 \mathrm{v}-\mathrm{Re} 1-\mathrm{F} 1$ & $93.14(7)$ \\
\hline $\mathrm{F} 1^{\mathrm{i}}-\mathrm{Re} 1-\mathrm{Cs} 1^{\mathrm{i}}$ & $53.533(6)$ \\
\hline $\mathrm{F} 1^{\mathrm{ii}}-\mathrm{Re} 1-\mathrm{Cs} 1^{\mathrm{i}}$ & $53.533(6)$ \\
\hline 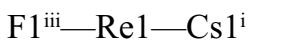 & $126.467(6)$ \\
\hline $\mathrm{F} 1^{\mathrm{iv}}-\mathrm{Re} 1-\mathrm{Cs} 1^{\mathrm{i}}$ & $124.74(6)$ \\
\hline $\mathrm{F} 1^{\mathrm{v}}-\mathrm{Re} 1-\mathrm{Cs} 1^{\mathrm{i}}$ & $55.26(6)$ \\
\hline $\mathrm{F} 1-\operatorname{Re} 1-\mathrm{Cs} 1^{\mathrm{i}}$ & $126.467(6)$ \\
\hline $\mathrm{F} 1^{\mathrm{i}}-\mathrm{Re} 1-\mathrm{Cs} 1^{\mathrm{vi}}$ & $124.74(6)$ \\
\hline $\mathrm{F} 1^{\mathrm{ii}}-\mathrm{Re} 1-\mathrm{Cs} 1^{\mathrm{vi}}$ & $53.533(6)$ \\
\hline 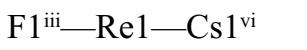 & $126.467(6)$ \\
\hline $\mathrm{F} 1^{\mathrm{iv}}-\mathrm{Re} 1-\mathrm{Cs} 1^{\mathrm{vi}}$ & $53.532(6)$ \\
\hline $\mathrm{F} 1^{\mathrm{v}}-\mathrm{Re} 1-\mathrm{Cs} 1^{\mathrm{vi}}$ & $126.468(6)$ \\
\hline $\mathrm{F} 1-\operatorname{Re} 1-\mathrm{Cs} 1^{\mathrm{vi}}$ & $55.26(6)$ \\
\hline $\mathrm{Cs} 1^{\mathrm{i}}-\mathrm{Re} 1-\mathrm{Cs} 1^{\mathrm{vi}}$ & $106.554(9)$ \\
\hline $\mathrm{F} 1^{\mathrm{i}}-\mathrm{Re} 1-\mathrm{Cs} 1^{\mathrm{vii}}$ & $55.26(6)$ \\
\hline $\mathrm{F} 1^{\mathrm{ii}}-\mathrm{Re} 1-\mathrm{Cs} 1^{\mathrm{vii}}$ & $126.467(6)$ \\
\hline 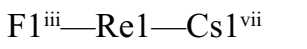 & $53.533(6)$ \\
\hline $\mathrm{F} 1^{\mathrm{iv}}-\mathrm{Re} 1-\mathrm{Cs} 1^{\mathrm{vii}}$ & $126.468(6)$ \\
\hline $\mathrm{F} 1^{\mathrm{v}}-\mathrm{Re} 1-\mathrm{Cs} 1^{\mathrm{vii}}$ & $53.532(6)$ \\
\hline $\mathrm{F} 1-\operatorname{Re} 1-\mathrm{Cs} 1^{\mathrm{vii}}$ & $124.74(6)$ \\
\hline 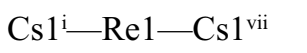 & $73.446(9)$ \\
\hline $\mathrm{Cs} 1^{\mathrm{vi}}-\mathrm{Re} 1-\mathrm{Cs} 1^{\mathrm{vii}}$ & 180.0 \\
\hline $\mathrm{F} 1 \mathrm{i}-\mathrm{Re} 1-\mathrm{Cs} 1$ & $126.467(6)$ \\
\hline $\mathrm{F} 1 \mathrm{ii}-\mathrm{Re} 1-\mathrm{Cs} 1$ & $126.467(6)$ \\
\hline $\mathrm{F} 1 \mathrm{iii}-\mathrm{Re} 1-\mathrm{Cs} 1$ & $53.533(6)$ \\
\hline $\mathrm{F} 1^{\mathrm{iv}}-\mathrm{Re} 1-\mathrm{Cs} 1$ & $55.26(6)$ \\
\hline $\mathrm{F} 1{ }^{\mathrm{v}}-\mathrm{Re} 1-\mathrm{Cs} 1$ & $124.74(6)$ \\
\hline $\mathrm{F} 1-\operatorname{Re} 1-\mathrm{Cs} 1$ & $53.533(6)$ \\
\hline $\mathrm{Cs} 1 \mathrm{i}-\mathrm{Re} 1-\mathrm{Cs} 1$ & 180.0 \\
\hline $\mathrm{Cs} 1^{\mathrm{vi}}-\mathrm{Re} 1-\mathrm{Cs} 1$ & $73.447(8)$ \\
\hline $\mathrm{Cs} 1{ }^{\mathrm{vii}}-\mathrm{Re} 1-\mathrm{Cs} 1$ & $106.553(8)$ \\
\hline $\mathrm{F} 1^{\mathrm{i}}-\mathrm{Re} 1-\mathrm{Cs} 1^{\mathrm{viii}}$ & $126.467(6)$ \\
\hline $\mathrm{F} 1^{\mathrm{ii}}-\mathrm{Re} 1-\mathrm{Cs} 1^{\mathrm{viii}}$ & $55.26(6)$ \\
\hline $\mathrm{F} 1^{\mathrm{iii}}-\mathrm{Re} 1-\mathrm{Cs} 1^{\mathrm{viii}}$ & $124.74(6)$ \\
\hline $\mathrm{F} 1^{\mathrm{iv}}-\mathrm{Re} 1-\mathrm{Cs} 1^{\mathrm{viii}}$ & $126.467(6)$ \\
\hline $\mathrm{F} 1^{\mathrm{v}}-\mathrm{Re} 1-\mathrm{Cs} 1^{\text {viii }}$ & $53.533(6)$ \\
\hline $\mathrm{F} 1-\mathrm{Re} 1-\mathrm{Cs} 1^{\mathrm{viii}}$ & $53.533(6)$ \\
\hline 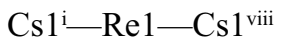 & $73.447(8)$ \\
\hline 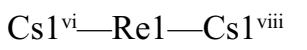 & $73.447(9)$ \\
\hline 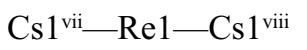 & $106.553(9)$ \\
\hline Cs1-Re1-Cs1 $1^{\text {vii }}$ & $106.553(9)$ \\
\hline 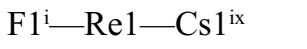 & $53.533(6)$ \\
\hline 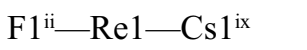 & $124.74(6)$ \\
\hline 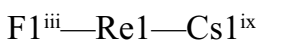 & $55.26(6)$ \\
\hline $\mathrm{F} 1^{\mathrm{iv}}-\mathrm{Re} 1-\mathrm{Cs} 1^{\mathrm{ix}}$ & $53.533(6)$ \\
\hline
\end{tabular}

\begin{tabular}{|c|c|}
\hline $\mathrm{F} 1^{\mathrm{xi}}-\mathrm{Cs} 1-\mathrm{F} 1^{\mathrm{xiv}}$ & $62.38(6)$ \\
\hline $\mathrm{F} 1^{\mathrm{x}}-\mathrm{Cs} 1-\mathrm{F} 1^{\mathrm{xiv}}$ & $97.70(3)$ \\
\hline $\mathrm{F} 1^{\mathrm{xii}}-\mathrm{Cs} 1-\mathrm{F} 1^{\mathrm{xiv}}$ & $129.122(17)$ \\
\hline $\mathrm{F} 1^{\mathrm{xiii}}-\mathrm{Cs} 1-\mathrm{F} 1^{\mathrm{xiv}}$ & $53.43(7)$ \\
\hline $\mathrm{F} 1^{\mathrm{xi}}-\mathrm{Cs} 1-\mathrm{F} 1^{\mathrm{iii}}$ & $97.70(3)$ \\
\hline $\mathrm{F} 1^{\mathrm{x}}-\mathrm{Cs} 1-\mathrm{F} 1^{\mathrm{iii}}$ & $129.122(17)$ \\
\hline 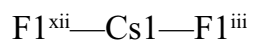 & $62.38(6)$ \\
\hline $\mathrm{F} 1^{\mathrm{xiii}}-\mathrm{Cs} 1-\mathrm{F} 1^{\mathrm{iii}}$ & $65.44(7)$ \\
\hline $\mathrm{F} 1^{\mathrm{xiv}}-\mathrm{Cs} 1-\mathrm{F} 1^{\mathrm{iii}}$ & $118.323(15)$ \\
\hline $\mathrm{F} 1^{\mathrm{xi}}-\mathrm{Cs} 1-\mathrm{F} 1^{\mathrm{xv}}$ & $97.70(3)$ \\
\hline $\mathrm{F} 1^{\mathrm{x}}-\mathrm{Cs} 1-\mathrm{F} 1^{\mathrm{xv}}$ & $62.38(6)$ \\
\hline $\mathrm{F} 1^{\mathrm{xii}}-\mathrm{Cs} 1-\mathrm{F} 1^{\mathrm{xv}}$ & $129.122(17)$ \\
\hline $\mathrm{F} 1^{\mathrm{xiii}}-\mathrm{Cs} 1-\mathrm{F} 1^{\mathrm{xv}}$ & $118.323(14)$ \\
\hline $\mathrm{F} 1^{\mathrm{xiv}}-\mathrm{Cs} 1-\mathrm{F} 1^{\mathrm{xv}}$ & $65.44(7)$ \\
\hline 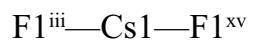 & $163.82(6)$ \\
\hline $\mathrm{F} 1{ }^{\mathrm{xi}}-\mathrm{Cs} 1-\mathrm{F} 1$ & $129.123(17)$ \\
\hline $\mathrm{F} 11^{\mathrm{x}}-\mathrm{Cs} 1-\mathrm{F} 1$ & $97.70(3)$ \\
\hline $\mathrm{F} 1^{\mathrm{xii}}-\mathrm{Cs} 1-\mathrm{F} 1$ & $62.38(6)$ \\
\hline $\mathrm{F} 1^{\mathrm{xii}}-\mathrm{Cs} 1-\mathrm{F} 1$ & $118.323(15)$ \\
\hline $\mathrm{F} 1^{\mathrm{xiv}}-\mathrm{Cs} 1-\mathrm{F} 1$ & $163.82(6)$ \\
\hline $\mathrm{F} 1^{\mathrm{iii}-\mathrm{Cs} 1-\mathrm{F} 1}$ & $53.43(7)$ \\
\hline $\mathrm{F} 1^{\mathrm{xv}}-\mathrm{Cs} 1-\mathrm{F} 1$ & $118.323(15)$ \\
\hline $\mathrm{F} 1^{\mathrm{xi}}-\mathrm{Cs} 1-\mathrm{F} 1^{\mathrm{xvi}}$ & $129.122(17)$ \\
\hline $\mathrm{F} 1^{\mathrm{x}}-\mathrm{Cs} 1-\mathrm{F} 1^{\mathrm{xvi}}$ & $62.38(6)$ \\
\hline $\mathrm{F} 1^{\mathrm{xii}}-\mathrm{Cs} 1-\mathrm{F} 1^{\mathrm{xvi}}$ & $97.70(3)$ \\
\hline $\mathrm{F} 1^{\mathrm{xiii}}-\mathrm{Cs} 1-\mathrm{F} 1^{\mathrm{xvi}}$ & $163.82(6)$ \\
\hline $\mathrm{F} 1^{\mathrm{xiv}}-\mathrm{Cs} 1-\mathrm{F} 1^{\mathrm{xvi}}$ & $118.323(15)$ \\
\hline 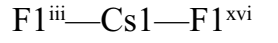 & $118.323(15)$ \\
\hline $\mathrm{F} 1^{\mathrm{xv}}-\mathrm{Cs} 1-\mathrm{F} 1^{\mathrm{xvi}}$ & $53.43(7)$ \\
\hline $\mathrm{F} 1-\mathrm{Cs} 1-\mathrm{F} 1^{\mathrm{xvi}}$ & $65.44(7)$ \\
\hline $\mathrm{F} 1^{\mathrm{xi}}-\mathrm{Cs} 1-\mathrm{F} 1^{\mathrm{xvii}}$ & $102.55(5)$ \\
\hline $\mathrm{F} 1^{x}-\mathrm{Cs} 1-\mathrm{F} 1^{\mathrm{xvii}}$ & $143.51(2)$ \\
\hline $\mathrm{F} 1^{\mathrm{xii}}-\mathrm{Cs} 1-\mathrm{F} 1^{\mathrm{xvii}}$ & $143.51(2)$ \\
\hline $\mathrm{F} 1^{\mathrm{xiii}}-\mathrm{Cs} 1-\mathrm{F} 1^{\mathrm{xvii}}$ & $49.86(5)$ \\
\hline $\mathrm{F} 1^{\mathrm{xiv}}-\mathrm{Cs} 1-\mathrm{F} 1^{\mathrm{xvii}}$ & $49.86(5)$ \\
\hline 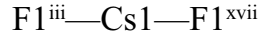 & $85.93(3)$ \\
\hline $\mathrm{F} 1^{\mathrm{xv}}-\mathrm{Cs} 1-\mathrm{F} 1^{\mathrm{xvii}}$ & $85.93(3)$ \\
\hline $\mathrm{F} 1-\mathrm{Cs} 1-\mathrm{F}^{\mathrm{xvii}}$ & $113.96(2)$ \\
\hline $\mathrm{F} 1^{\mathrm{xvi}}-\mathrm{Cs} 1-\mathrm{F} 1^{\mathrm{xvi}}$ & $113.96(2)$ \\
\hline $\mathrm{F} 1^{\mathrm{xi}}-\mathrm{Cs} 1-\mathrm{F} 1^{\mathrm{iv}}$ & $143.51(2)$ \\
\hline $\mathrm{F} 1^{\mathrm{x}}-\mathrm{Cs} 1-\mathrm{F} 1^{\mathrm{iv}}$ & $143.51(2)$ \\
\hline $\mathrm{F} 1^{\mathrm{xii}}-\mathrm{Cs} 1-\mathrm{F} 1^{\mathrm{iv}}$ & $102.55(5)$ \\
\hline $\mathrm{F} 1^{\mathrm{xiii}}-\mathrm{Cs} 1-\mathrm{F} 1^{\mathrm{iv}}$ & $85.93(3)$ \\
\hline $\mathrm{F} 1^{\mathrm{xiv}}-\mathrm{Cs} 1-\mathrm{F} 1^{\mathrm{iv}}$ & $113.96(2)$ \\
\hline $\mathrm{F} 1^{\mathrm{iii}}-\mathrm{Cs} 1-\mathrm{F} 1^{\mathrm{iv}}$ & $49.86(5)$ \\
\hline $\mathrm{F} 1^{\mathrm{xv}}-\mathrm{Cs} 1-\mathrm{F} 1^{\mathrm{iv}}$ & $113.96(2)$ \\
\hline $\mathrm{F} 1-\mathrm{Cs} 1-\mathrm{F} 1^{\mathrm{iv}}$ & $49.86(5)$ \\
\hline $\mathrm{F} 1^{\mathrm{xvi}}-\mathrm{Cs} 1-\mathrm{F} 1^{\mathrm{iv}}$ & $85.93(3)$ \\
\hline
\end{tabular}




\begin{tabular}{|c|c|}
\hline $\mathrm{F} 1^{\mathrm{v}}-\operatorname{Re} 1-\mathrm{Cs} 1^{\mathrm{ix}}$ & $126.467(6)$ \\
\hline $\mathrm{F} 1-\operatorname{Re} 1-\mathrm{Cs} 1^{\mathrm{ix}}$ & $126.467(6)$ \\
\hline $\mathrm{Cs} 11^{\mathrm{i}}-\operatorname{Re} 1-\operatorname{Cs} 1^{\mathrm{ix}}$ & $106.553(8)$ \\
\hline 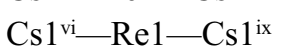 & $106.553(9)$ \\
\hline 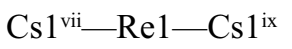 & $73.447(9)$ \\
\hline $\operatorname{Cs} 1-\operatorname{Re} 1-\operatorname{Cs} 1^{\text {ix }}$ & $73.447(9)$ \\
\hline $\mathrm{Cs} 1^{\mathrm{viii}}-\operatorname{Re} 1-\mathrm{Cs} 1^{\mathrm{ix}}$ & 180.0 \\
\hline $\mathrm{Re} 1-\mathrm{F} 1-\mathrm{Cs} 1^{\mathrm{x}}$ & $162.67(9)$ \\
\hline $\mathrm{Re} 1-\mathrm{F} 1-\mathrm{Cs} 1$ & $96.61(3)$ \\
\hline $\mathrm{Cs} 1^{\mathrm{x}}-\mathrm{F} 1-\mathrm{Cs} 1$ & $82.30(3)$ \\
\hline $\operatorname{Re} 1-\mathrm{F} 1-\mathrm{Cs} 1^{\mathrm{viii}}$ & $96.61(3)$ \\
\hline $\mathrm{Cs} 1^{\mathrm{x}}-\mathrm{F} 1-\mathrm{Cs} 1^{\mathrm{viii}}$ & $82.30(3)$ \\
\hline
\end{tabular}

$\begin{array}{ll}\mathrm{F} 1^{\mathrm{xvi}}-\mathrm{Cs} 1-\mathrm{F} 1^{\mathrm{iv}} & 64.10(5) \\ \mathrm{F} 1^{\mathrm{xi}}-\mathrm{Cs} 1-\mathrm{F} 1^{\mathrm{vi}} & 143.51(2) \\ \mathrm{F} 1^{\mathrm{x}}-\mathrm{Cs} 1-\mathrm{F} 1^{\mathrm{vi}} & 102.55(5) \\ \mathrm{F} 1^{\mathrm{xii}}-\mathrm{Cs} 1-\mathrm{F} 1^{\mathrm{vi}} & 143.51(2) \\ \mathrm{F} 1^{\mathrm{xii}}-\mathrm{Cs} 1-\mathrm{F} 1^{\mathrm{vi}} & 113.96(2) \\ \mathrm{F} 1^{\mathrm{xi}}-\mathrm{Cs} 1-\mathrm{F} 1^{\mathrm{vi}} & 85.93(3) \\ \mathrm{F} 1^{\mathrm{iii}}-\mathrm{C} 1-\mathrm{F} 1^{\mathrm{vi}} & 113.96(2) \\ \mathrm{F} 1^{\mathrm{xv}}-\mathrm{Cs} 1-\mathrm{F} 1^{\mathrm{vi}} & 49.86(5) \\ \mathrm{F} 1-\mathrm{Cs} 1-\mathrm{F} 1^{\mathrm{vi}} & 85.93(3) \\ \mathrm{F} 1^{\mathrm{xv}}-\mathrm{Cs} 1-\mathrm{F} 1^{\mathrm{vi}} & 49.86(5) \\ \mathrm{F} 1^{\mathrm{xvi}}-\mathrm{Cs} 1-\mathrm{F} 1^{\mathrm{vi}} & 64.10(5) \\ \mathrm{F} 1^{\mathrm{iv}}-\mathrm{Cs} 1-\mathrm{F} 1^{\mathrm{vi}} & 64.10(5)\end{array}$

Symmetry codes: (i) $-x,-y,-z$; (ii) $y,-x+y,-z$; (iii) $-y, x-y, z$; (iv) $x-y, x,-z$; (v) $-x+y,-x, z$; (vi) $-x+1,-y+1,-z$; (vii) $x-1, y-1, z$; (viii) $x, y-1, z$; (ix) $-x,-y+1,-z$; (x) $-x+1,-y+1,-z+1$; (xi) $y,-x+y+1,-z+1$; (xii) $x-y, x,-z+1$; (xii) $-x+y,-x+1, z$; (xiv) $x, y+1, z$; (xv) $-y+1, x-y+1, z$; (xvi) $-x+y+1,-x+1$, $z$; (xvii) $y,-x+y+1,-z$. 